\title{
Inhibition of Metabotropic Glutamate Receptor III facilitates sensitization to alkylating chemotherapeutics in Glioblastoma
}

\section{Julian Maier}

University Clinic of Freiburg https://orcid.org/0000-0002-2957-9031

\section{Vidhya Ravi}

University Clinic of Freiburg

Jan Kueckelhaus

University Clinic of Freiburg

\section{Simon Behringer}

University Clinic of Freiburg

\section{Niklas Garrelfs}

University Clinic of Freiburg

\section{Paulina Will}

University Clinic of Freiburg https://orcid.org/0000-0001-5924-2432

\section{Na Sun}

Helmholtz Zentrum München

Jasmin Ehr

University Clinic of Freiburg

Jonathan Goeldner

Microenvironment and Immunology Research Laboratory, Medical Center, University of Freiburg,

Germany https://orcid.org/0000-0003-2394-7312

\section{Dietmar Pfeifer}

University Clinic of Freiburg

\section{Marie Follo}

University Medical Center

\section{Luciana Hannibal}

University Clinic of Freiburg

\section{Axel Walch}

Helmholtz Zentrum München

\section{Ulrich Hofmann}

University Clinic of Freiburg https://orcid.org/0000-0002-6264-3701

\section{Jürgen Beck}

University Clinic of Freiburg 


\section{Dieter Henrik Heiland}

Medical Center - University of Freiburg https://orcid.org/0000-0002-9258-3033

\section{Oliver Schnell}

University Clinic of Freiburg

Kevin Joseph ( $\nabla$ kevin.joseph@uniklinik-freiburg.de )

University Clinic of Freiburg https://orcid.org/0000-0001-6317-8736

\section{Article}

Keywords: Glioblastoma (GBM), central nervous system, metabotropic glutamate receptor III

Posted Date: June 2nd, 2021

DOl: https://doi.org/10.21203/rs.3.rs-337223/v1

License: (c) (1) This work is licensed under a Creative Commons Attribution 4.0 International License.

Read Full License

Version of Record: A version of this preprint was published at Cell Death \& Disease on July 21st, 2021. See the published version at https://doi.org/10.1038/s41419-021-03937-9. 


\section{Abstract}

Glioblastoma (GBM), the most malignant tumor of the central nervous system, is marked by its dynamic response to microenvironmental niches. In particular, this cellular plasticity contributes to the development of an immediate resistance during tumor treatment. Novel insights into the developmental trajectory exhibited by GBM show a strong capability to respond to its microenvironment by clonal selection of specific phenotypes. Using the same mechanisms, malignant GBM do develop intrinsic mechanisms to resist chemotherapeutic treatments. This resistance was reported to be sustained by the paracrine and autocrine glutamate signaling via ionotropic and metabotropic receptors. However, the extent to which glutamatergic signaling modulates the chemoresistance and transcriptional profile of the GBM remains unexplored. In this study we aimed to map the manifold effects of glutamate signaling in GBM as the basis to further discover the regulatory role and interactions of specific receptors, within the GBM microenvironment. Our work provides insights into glutamate release dynamics, representing its importance for GBM growth, viability, and migration. Based on newly published multi-omic datasets, we explored the and characterized the functions of different ionotropic and metabotropic glutamate receptors, of which the metabotropic receptor 3 (GRM3) is highlighted through its modulatory role in maintaining the ability of GBM cells to evade standard alkylating chemotherapeutics. We addressed the clinical relevance of GRM3 receptor expression in GBM and provide a proof of concept where we manipulate intrinsic mechanisms of chemoresistance, driving GBM towards chemo-sensitization through GRM3 receptor inhibition. Finally, we validated our findings in our novel human organotypic sectionbased tumor model, where GBM growth and proliferation was significantly reduced when GRM3 inhibition was combined with temozolomide application. Our findings present a new picture of how glutamate signaling via mGluR3 interacts with the phenotypical GBM transcriptional programs in light of recently published GBM cell-state discoveries.

\section{Introduction}

There is a rising interest in the metabolic imbalances that are present within the glioblastoma environment, with recent reports highlighting the interface between GBM cells and neural cells within the local microenvironment, driving invasion and proliferation by means of glutamatergic signaling $(1,2)$. In addition to glutamatergic inputs from the neuronal environment, GBM has been shown to release glutamate in the nearby environment, interacting with $\mathrm{Ca}^{2+}$ permeable AMPA receptors, promoting cellular migration and proliferation through Akt activation (3-6). The increased level of glutamate in the brain also has clinical relevance, with patients suffering from GBM showing increased cortical levels of glutamate, claimed to be responsible for seizure and excitotoxicity in cells close to the tumor site (7). In healthy neuronal cells, the mGLuR's have been shown to control the postsynaptic neuronal response to glutamate, modulating ionotropic glutamate receptor activity, leading to cellular proliferation, growth, migration, survival, and calcium-mediated cellular homeostasis $(8,9)$. Besides the reported significance of ionotropic glutamate receptors, the role of metabotropic glutamate receptors (mGluR's) remains a topic of interest in GBM. 
In this work, we demonstrate that glutamate uptake/release is necessary for GBM survival, with glutamate levels being dynamically modulated by cellular stress, validated within a spatial multi-omic dataset. We identified the expression of both ionotropic and metabotropic receptors from a GBM development context and characterized the role each plays in modulating cellular kinetics and proliferation. This enabled us to identify and further characterize the expression of metabotropic glutamate receptor 3 (GRM3) in clinical samples, presenting evidence that GRM3 expression was significantly increased and of potential interest in recurrent GBM patients. We report that inhibition of mGluR3 at a non-toxic concentration of 60-100 nM was enough to drive chemotherapeutic resistant GBM to a transcriptional state that resulted in reduced network forming characteristics, which enabled targeted action by means of temozolomide. These results were further validated in our previously published human organotypic section-based tumor model, where tumor growth and proliferation were observed to be significantly reduced when mGluR3 inhibition was carried out in conjunction with temozolomide administration.

\section{Materials And Methods}

\section{Ethical Approval}

The local ethics committee of the University of Freiburg approved data evaluation, imaging procedures and experimental design (protocol 1000020/09 and 472/15_160880). All methods were carried out in accordance with the approved guidelines. Written informed consent was obtained from all patients. The studies were approved by an institutional review board. Further information and requests for resources, raw data and reagents should be directed and will be fulfilled by either by $\mathrm{K}$. Joseph, kevin.joseph@uniklinik-freiburg.de or D.H. Heiland, dieter.henrik.heiland@uniklinik-freiburg.de.

\section{Cell Culture}

Three glioblastoma cell lines, CLGSC, CL168 and CL233, were purified from surgical specimens with the informed consent of patients as described previously by our research group (10). The obtained cell suspensions were then cultured in Minimum Essential Medium (MEM) culture medium (21090022, Thermo Fisher Scientific Inc., Waltham, MA, USA) supplemented with $10 \%$ Fetal-calf serum (FCS) (P303306; PAN-Biotech GmbH, Aidenbach, Germany) and 1\% Penicillin/Streptomycin (15140122, Thermo Fisher Scientific Inc., Waltham, MA, USA). Cell viability and confluence were monitored regularly (48-72 hours) by microscopic observation and the culture medium was changed every 5-7 days.

\section{Viral Transduction by Constitutive Reporter Lentiviral Vectors}

For live imaging and whole-cell tracking, primary cultured glioblastoma cells were transduced with lentiviral particles (rLV.EF1.ZsGreen1-9 and rLV.EF1.mCherry-9

0037VCT, 0038VCT Takara Bio, Kusatsu, Japan) as previously described (11-13). Briefly, $5 \times 10^{5}$ cells were seeded on a round petri dish. Viral particle quantity was calculated according to the manufacturer's 
instructions and the transduction mix was prepared by adding the required volume of thawed viral particles and Polybrene ${ }^{\circledR}(10 \mu \mathrm{g} / \mathrm{ml}$, TR-1003-G, Merck KGaA, Darmstadt, Germany). Cells were then incubated overnight in the transduction mix at $37^{\circ} \mathrm{C}, 5 \% \mathrm{CO}_{2}$. Efficacy and quality of transduction was microscopically evaluated after 48 hours.

\section{Glutamate monitoring}

Extra- and intracellular glutamate concentrations were determined by using the Glutamate Assay Kit (ab83389, Abcam, Cambridge, UK or MAK004, Sigma-Aldrich, St. Louis, MO, USA) according to the manufacturer's instructions. For extracellular glutamate concentrations, $50 \mu \mathrm{l}$ from either cell culture or human organotypic slice cultures was used. Samples were snap-frozen in liquid nitrogen or directly analyzed according to the manufacturer's protocol. For intracellular glutamate measurements, a dounce glass potter homogenizer was used for mechanical lysis of the cells. As both assays are based on a colorimetric detection method, the amount of glutamate was quantified by measuring the absorbance at $450 \mathrm{~nm}$ using a microplate reader (TECAN infinite M200, Zurich, Switzerland). Glutamate concentrations were calculated according to the manufacturer's calculation guide and stated in $\mu \mathrm{M}$. For every experiment a glutamate standard curve and background measurements of culture medium and control groups were calculated and taken into account for individual glutamate calculations.

$$
\text { Sa }=\left(\frac{\text { Corrected absorbance }-(y-\text { intercept })}{\text { Slope }}\right)
$$

$$
\text { Glutamate Concentration }=\left(\frac{S a}{S v}\right) * D
$$

$\mathrm{Sa}=$ Amount of sample $(\mathrm{nmol})$ from standard curve

Sv $=$ Volume of sample $(\mu \mathrm{L})$ added into the well

$D=$ Sample dilution factor.

\section{Mass Spectroscopy (LC-MS)}

Materials. All materials utilized for the targeted analysis of neurotransmitters in tissue biopsies are listed in Table 1.

\section{Methods}

LC-MS/MS Method. The separation and detection of metabolites was performed according to the overall strategy of Xu et al ${ }^{1}$, with modifications. Metabolites were separated on a AC 3 AQ column, $1.0 \times 150 \mathrm{~mm}$ (HiChrom) using a gradient of solvents $A(0.1 \%$ formic acid in water) and $B(0.1 \%$ formic acid in $\mathrm{MeOH})$ over 10 minutes as follows: $0-0.5$ min $15 \%$ B, 0.5-5 min $85 \%$ B, 5-6 min $85 \%$ B, 6-6.20 min $15 \%$ B, 6.21$10 \mathrm{~min} 15 \% \mathrm{~B}$. The flow rate was set to $0.050 \mathrm{~mL} / \mathrm{min}$ connected to a Nexera X2 LC system (Shimadzu). 
Metabolites were detected on a Sciex 6500+ ESI-tripleQ MS/MS on low mass mode (0-1000 Da), with curtain gas (CUR) at 40 , collision gas (CAD) at 10 , lon spray voltage (IS) at 5000 Volts, temperature (TEM) at $400{ }^{\circ} \mathrm{C}$, ion source gas 1 (GS1) at 40 and ion source gas 2 (GS2) at 30. Each metabolite was optimized individually using chromatographic solvent conditions and exhibited optimal ionization in the positive mode (Table 2).

Calibrators and internal standards. All metabolites were quantified with respect to a calibration curve and selected internal standards. The dynamic range of calibrators was $0-5 \mu \mathrm{M}$ for Glutamate, Serine, dopamine, serotonin and GABA, and 0-100 $\mu \mathrm{M}$ for Glycine, Tryptophan, Tyrosine, Choline and 2-LAminoadipic acid. D4- dopamine was utilized as internal standard for the quantification of dopamine. Glycine- $-1{ }^{13} \mathrm{C},{ }^{15} \mathrm{~N}$ was utilized as internal standard for the quantification of Glycine. All other metabolites were normalized by isoprenaline as the internal standard, as described in a published method validated according to FDA guidelines (14). The internal standard mix contained D4-dopamine (5 $\mu \mathrm{M})$, Glycine-1${ }^{13} \mathrm{C},{ }^{15} \mathrm{~N}(50 \mu \mathrm{M})$ and isoprenaline $(50 \mu \mathrm{M})$.

\section{Sample handling.}

Tissue biopsies: Flash-Frozen tissue biopsies were stored at $-80^{\circ} \mathrm{C}$. On the day of sample preparation, the frozen tissue was weighed quickly by placing it on a small piece of aluminum foil. The tissue was thawed on ice and resuspended with ice-cold lysis buffer (10 mL PBS supplemented with $100 \mu \mathrm{L}$ protease inhibitor cocktail (P8340-5ML, Sigma-Aldrich, St. Louis, MO, USA)) to a ratio of $100 \mathrm{mg}$ tissue per $\mathrm{mL}$ of lysis buffer. The tissue was homogenized on ice with a tissue disruptor (47747-370, VWR, Radnor, PA, US) until the mixture was fully homogeneous. An aliquot of this crude extract was diluted 1:10 with lysis buffer and utilized for metabolomics analysis and protein quantification by the bicinchoninic acid assay (Pierce BCA Protein Assay Kit (23225), Thermofisher Scientific, USA). The remaining undiluted tissue extract was frozen quickly in dry-ice and stored at $-80^{\circ} \mathrm{C}$.

Sample preparation: Neurotransmitters were extracted according to a fast acidic methanol extraction procedure previously described for polar metabolite extraction (15). Briefly, $20 \mu \mathrm{L}$ of calibrator or biological sample of interest was mixed with $20 \mu \mathrm{L}$ of internal standard mix and $20 \mu \mathrm{L}$ of $0.1 \%$ formic acid in $\mathrm{H}_{2} \mathrm{O}$. Metabolites were extracted by addition of $100 \mu \mathrm{L}$ of $0.1 \%$ formic acid in $\mathrm{MeOH}$. Samples were vortexed, then centrifuged at $9447 \times \mathrm{g}$ at room temperature for 10 minutes and the supernatants were transferred into HPLC vials.

Data analysis. Quantification of metabolites was carried out with Analyst ${ }^{\circledR} 1.6 .3$ software, 2015 AB Sciex.

\section{Cell Proliferation ELISA, BrdU (colorimetric)}

For the assessment of cell proliferation, the colorimetric BrdU ELISA immunoassay by Roche (11647229001; Roche, Basel, Switzerland) was used according to the manufacturer's instructions. This method is based on the measurement of BrdU incorporation during DNA-synthesis in replicating cells. For 
all Cell Proliferation ELISA, BrdU (colorimetric) experiments, $5 \times 10^{3}$ cells were seeded per well $(100 \mu \mathrm{l})$ on 96-well culture plates (655180; Greiner Bio-One International GmbH, Kremsmünster, Austria). For every experiment, triplets of all different conditions and at least one untreated control group were set up. Cells were incubated for 48 hours at $37^{\circ} \mathrm{C}, 5 \% \mathrm{CO}_{2}$ to ensure satisfying cell viability and confluency before the treatment solutions were administered. Treatment periods were terminated by replacing the treatment solutions with MEM culture medium, followed by 6 hours BrdU incorporation with BrdU labeling solution according to the manufacturer's instructions. Colorimetric measurements were performed after the addition of $25 \mu \mathrm{l} 1 \mathrm{M} \mathrm{H}_{2} \mathrm{SO}_{4}$ (stop solution) to each well and $1 \mathrm{~min}$ incubation on a shaker at $300 \mathrm{rpm}$. A microplate reader (TECAN infinite M200, Zurich, Switzerland) measured the absorbance at $450 \mathrm{~nm}$ in reference to wavelength $690 \mathrm{~nm}$.

\section{Cell Proliferation and Cytotoxicity Assay (WST-8)}

Cell Proliferation and cytotoxicity was assessed by using the Cell Counting Kit 8 (WST-8) (ab228554, Abcam, Cambridge, UK). This method is based on the colorimetric change of WST-8 / CCK tetrazolium salt when reduced to an orange formazan dye by cellular dehydrogenases. Briefly, cells were seeded at a density of $5 \times 10^{3}$ cells per well $(100 \mu \mathrm{l})$ on 96-well plates $(655180$, Greiner Bio-One International GmbH, Kremsmünster, Austria) and incubated for 48 hours at $37^{\circ} \mathrm{C}, 5 \% \mathrm{CO}_{2}$ to ensure satisfying cell viability and confluency. Cells were then treated for usually 24 hours at $37^{\circ} \mathrm{C}, 5 \% \mathrm{CO}_{2}$, before the WST-8 assay was performed. After termination of the treatment period, all treatment solutions were discarded and replaced. According to the manufacturer's instructions, $10 \mu \mathrm{l}$ WST-8-solution was added to each well, followed by 4 hours incubation at $37^{\circ} \mathrm{C}, 5 \% \mathrm{CO}_{2}$, protected from light. Absorbance was read at a wavelength of $460 \mathrm{~nm}$ using a microplate reader (TECAN infinite M200, Zurich, Switzerland).

\section{IncuCyte S3 Live-Cell Analysis}

The IncuCyte S3 Live-Cell Analysis System (Essen BioScience Inc., Sartorius AG, Göttingen, Germany) was used for image-based analyses and whole cell-tracking of glioblastoma cell lines under different treatment conditions in a time-dependent manner. By using ZsGreen and mCherry transfected GBM cell lines, it was possible to investigate individual cell proliferation by cell count statistics, as well as profiling cellular activity and migration. Therefore, $1,5 \times 10^{3}$ cells per well were seeded on 96 -well plates (655180; Greiner Bio-One International $\mathrm{GmbH}$, Kremsmünster, Austria) and incubated for 48 hours at $37^{\circ} \mathrm{C}, 5 \% \mathrm{CO}_{2}$, to ensure satisfying cell viability and confluency. Thereafter, cells were treated with different treatment solutions and incubated at $37^{\circ} \mathrm{C}, 5 \% \mathrm{CO}_{2}$. Fluorescence images were acquired in various time-intervals (10 min $-3 \mathrm{~h}$ ) for a total time period of up to 3 days. Optical modules were set for up to two channels, green (acquisition time $100 \mathrm{~ms}$ ) and red (acquisition time $100 \mathrm{~ms}$ ), using the 10x objective. The obtained data were processed with the device-specific software (IncuCyte 2019B) to precisely determine individual cell counts. Additional migration and activity tracking were performed using CellTracker software and post-processed by celltracer $\mathrm{R}$ software package. 
Abcam's Kinetic Apoptosis Kit (Microscopy) (AB129817, Abcam, Cambridge, UK) was used for the visual assessment of early and late apoptotic events. The assay is based on PSIVA ${ }^{\mathrm{TM}}$, an Annexin XII-based polarity sensitive probe which is conjugated to IANBD, a polarity sensitive dye that fluoresces only when bound to phosphatidyl-serine in the non-polar environment of the membrane lipid bilayer of the cells. The pSIVA-IANBD (green) based assay was used in combination with propidium iodide $(\mathrm{PI}, \mathrm{red})$ to distinguish between non-apoptotic (pSIVA-IANBD negative/PI negative), early apoptotic (pSIVA-IANBD positive/PI negative), and late apoptotic or necrotic cells (pSIVA-IANBD positive/PI positive), respectively. According to the manufacturer's instructions, $20 \mu \mathrm{l}$ of pSIVA-IANBD and $10 \mu \mathrm{l}$ of PI per $1,5 \mathrm{ml}$ of culture medium were used. Microscopic imaging was performed by using the IncuCyte S3 Live-Cell Analysis System as described in the previous section.

\section{Cellular kinetics analysis}

The images obtained using the IncuCyte S3 Live-Cell Analysis were loaded into the image software CellTracker (16) and underwent vignetting correction and automatic alignment. Subsequently cells were tracked semi-automatically. This option requires the user to manually tag all cells that are supposed to be tracked in the first image. The software than proceeds with the tagged cells and tracks them over all images. The 'Timepoint data' was kept and subsequently processed using the R-Software package 'celltracer' (https://github.com/kueckelj/celltracer). The celltracer-object was set up with the function celltracer::initiateCTO(). Only cells that were tracked from the first till at least the second last image were included in downstream analysis. Cells that did not meet this quality requirement were discarded. A total of 9867 cells were analyzed. Figures were plotted using the following functions:

Fig. 4e, 5c - celltracer::plotAllTracks()

Supp. Fig. 4.d, Supp. Fig. 5.a - celltracer:::plotVelocityHeatmap()

Supp. Fig. 4.f, Supp. Fig. 5.b - celltracer::plotBoxplot0

Supp. Fig. 4.e, Supp. Fig. 5.c - celltracer::plotRidgeplot()

\section{Human Organotypic Slice Model}

\section{Pre-sectioning preparation}

To avoid any contamination of the tissue during the cortical sectioning, a sterile working field was set up for the whole sectioning procedure. All tools required for the procedure were sterilized following clinical hygiene protocols and placed within reach of the experimenter to prevent any delays. A vibratome (VT1200; Leica Biosystems) was used to prepare the sections. To maintain the best-possible tissue environment, the sectioning chamber was filled with ice-cold preparation medium (see Supp. Table 3. Preparation Medium Human Organotypic Slice Model), saturated with carbogen $\left(95 \% \mathrm{O}_{2}\right.$ and $\left.5 \% \mathrm{CO}_{2}\right)$. Millicell ${ }^{\circledR}$ cell culture inserts (PICM0RG50, Merck KGaA, Darmstadt, Germany) were placed in each well of six-well culture plates and filled with $1 \mathrm{ml}$ of growth medium. 


\section{Preparation of Human Organotypic Brain Slices}

All tissue blocks were directly transferred from the OR to the laboratory and submerged in preparation medium. Subsequently, all visibly damaged areas of the tissue were dissected away. After mounting the processed tissue block on the vibratome, $300-\mu \mathrm{m}$-thick sections were obtained at $0.12 \mathrm{~mm} / \mathrm{s}$ and were incubated in cold preparation medium $\left(4^{\circ} \mathrm{C}\right)$ for 10 min before plating on Millicell ${ }^{\circledR}$ Cell Culture Inserts. The entire growth medium was replaced 24 hours post-plating and every 48 hours thereafter. From each donor sample, three to four sections were freshly fixed in 4\% PFA post sectioning and used as control specimens. At the terminal time point, sections were fixed in 4\% PFA for further immunofluorescencebased staining.

\section{Tumor injection onto tissue cultures}

For monitoring the tumor invasion and proliferation profile in the brain sections, ZsGreen transfected glioblastoma cell lines (CLGSC, CL233) were used. Therefore, the ZsGreen transfected GBM cell lines were trypsinized and counted by using a hemocytometer. Post-trypsinization, $1 \times 10^{6}$ cells were harvested, centrifuged (200xg; RT; $5 \mathrm{~min}$ ) and resuspended in $50 \mu \mathrm{l}$ PBS to a final concentration of $20.000 \mathrm{cells} / \mu \mathrm{l}$. The obtained cell suspension was immediately used for tumor injection onto the brain section. For the injection, a $10 \mu \mathrm{l}$ Hamilton syringe (80330; Hamilton Company, Bonaduz, Switzerland) was used to manually inject approximately $1 \mu \mathrm{l}$ of the GBM cell suspension into the white-grey matter interface in the sections. Sections with injected GBM tumor cells were incubated at $37^{\circ} \mathrm{C}, 5 \% \mathrm{CO}_{2}$, and fresh culture medium was added every 24-48 hours. Tumor proliferation was monitored by imaging at day 0, 2 postinjection (DPI) and day 2, 4 and 6 post-treatment (DPT) by using an inverted fluorescence microscope (Zeiss, Observer D.1).

\section{Immunofluorescence (IF)}

Sections were washed three times with PBS for 5 minutes each and fixed in $20 \%$ methanol solution for 5 minutes at room temperature. After another three washing cycles with PBS, the sections were incubated overnight at $4^{\circ} \mathrm{C}$ in $1 \%$ Triton solution. The sections were then washed twice with PBS and subsequently blocked with a Triton-BSA (20\% BSA+0.1\% Triton X-100) blocking solution for 4 hours at room temperature. After another three PBS wash cycles, the primary antibodies were diluted in a Triton-BSA (1:500, 5\% BSA+0.1\% Triton X-100) mix and incubated overnight at $4^{\circ} \mathrm{C}$. All sections were then washed twice with PBS before the sections were incubated with secondary antibodies (1:1000,0.1\% Triton+5\% BSA) for 2 hours at room temperature. Finally, the sections were washed twice and transferred to microscopic slides. For all primary and secondary antibodies, appropriate concentrations were used according to the manufacturer's instructions as indicated in Supp. Table 1-2. Fluorescence microscopy was performed by using the Fluoview FV10i confocal microscope from Olympus. All measurements and image processing were performed using the company's software and FIJI (17).

\section{Immunohistochemistry (IHC)}


For immunohistochemical analysis, 30 samples of 18 patients with available de-novo (R0), first recurrence (R1) or paired samples of R0 and R1 tissue samples were selected. All samples were sourced from patients diagnosed with WHO Grade IV Glioblastoma (GBM). Tissue samples were fixed in $4 \%$ formaldehyde and paraffin-embedded according to standard procedures as described previously (18). Immunohistochemistry Application Solutions Kit (Rabbit) (13079S, Cell Signaling Technology, Danvers, USA) was used for all immunohistochemical staining. Deparaffinization and heat-induced epitope retrieval (HIER) in citrate buffer of $3 \mu \mathrm{m}$ paraffin-embedded tissue sections were performed according to the manufacturer's instructions. Sections were incubated overnight at $4^{\circ} \mathrm{C}$ in anti-GRM3 antibody (ab188750, 1:250, Abcam, Cambridge, UK). The sections were then incubated in SignalStain Boost Solution and Secondary Antibody Solution (DAB-Solution), followed by counterstaining with Meyer's haemalaun solution and mounted on glass slides. Imaging was carried out using an Olympus BX40 microscope. GRM3 positive cells of 4-6 optical fields (20x magnification) were counted per slide, and the mean number of GRM3 positive cells per $\mu \mathrm{m}^{2}$ was calculated.

\section{Statistical Analysis of Clinical Data}

For analysis of clinical data, a Cox Regression model was used. Kaplan Meier Survival plots were built by the survival package in R software environment (19). The ranked plots of IHC GRM3 protein expression and survival were plotted using a custom function implemented in R. Code available on reasonable request.

\section{Microarray Gene Expression Analysis}

For Microarray Gene Expression Analysis, all glioblastoma cell lines used in this study were profiled. Biological independent triplets were set up for all conditions, with 200.000 cells resuspended in $5 \mathrm{ml}$ of MEM culture medium and cultured in $25 \mathrm{~cm}^{2}$ cell culture flasks at $37^{\circ} \mathrm{C}, 5 \% \mathrm{CO}_{2}$. For treatment conditions, cells were treated with freshly prepared treatment solutions according to the manufacturer's instructions. Based on previous results of cell viability and apoptosis analysis by the Kinetic Apoptosis Kit, cells were treated for 4 hours at $37^{\circ} \mathrm{C}, 5 \% \mathrm{CO}_{2}$. Thereafter, the supernatant of every sample was transferred to $15 \mathrm{ml}$ falcon tubes to prevent any cell loss in the case of cell-detachment during the treatment period. The cell culture flasks were washed with $2 \mathrm{ml}$ of PBS, which was also collected in $15 \mathrm{ml}$ falcon tubes. Remaining adherent cells were enzymatically detached by $1 \mathrm{ml}$ Trypsin $0,25 \%$ for $2-3 \mathrm{~min}$ at $37^{\circ} \mathrm{C}, 5 \% \mathrm{CO}_{2}$. The enzymatic reaction was stopped by the addition of $3 \mathrm{ml} \mathrm{MEM}$ culture medium containing $10 \%$ FCS and the cell suspension was transferred to the $15 \mathrm{ml}$ falcon tubes.

\section{RNA extraction and isolation}

The collected cell suspensions were centrifuged for $10 \mathrm{~min}$ at $3000 \mathrm{xg}$ and the supernatant was discarded. Subsequent RNA extraction and isolation was performed by using the PicoPure RNA Isolation Kit (KIT0204,Thermo Fisher Scientific Inc., Waltham, MA, USA) for RNA Extraction according to the manufacturer's instructions. To avoid any RNase contamination, only new certified nucleic acid-free 
plasticware, as well as only new, sterile, RNase-free pipette tips and microcentrifuge tubes were used throughout the experiment. To avoid the introduction of RNase, all work surfaces were cleaned with RNase decontamination solution.

\section{RNA quantification and quality assessment}

For the accurate quantification of our RNA sample concentrations, the Invitrogen Qubit RNA BR (broad range) Assay Kit (Q10210; Thermo Fisher Scientific Inc., Waltham, MA, USA) was used according to the manufacturer's instructions. Concentration differences between samples were equalized by dilution with elution buffer to a consistent RNA concentration (100 ng / $3 \mu \mathrm{l}$ ) of each sample. Additionally, sample quality was controlled by performing the Bioanalyzer RNA 6000 Nano assay with RNA Nano Chips and the 2100 Bioanalyzer instrument according to the manufacturer's instruction, and subsequent analysis with 2100 Expert Software (Agilent Technologies, Santa Clara, CA, US).

\section{Reverse Transcription and Microarray Transcriptome Profiling}

The Clariom ${ }^{\mathrm{TM}}$ D Assay, human (902925 Clariom D Pico Assay, human, Thermo Fisher Scientific Inc., Waltham, USA) was used for RNA to cDNA conversion and Microarray Transcriptome Profiling and was carried out using an Affymetrix GS3000 GeneChip System.

\section{Microarray Gene Expression Analysis}

Data from microarray sequencing was background corrected and scaled using the LIMMA package, in the R computing environment. Differential gene expression analysis was carried out using DeSeq2 (20). Further analysis and plots were generated out using our inhouse VisLab package (heilandd/Vis_Lab1.5:

Vis_Lab1.5 (github.com)) Gene expression networks were generated using the clusterprofiler package in R (21).

\section{Results}

\section{Glutamate enrichment in the Glioblastoma microenvironment}

The presence of increased glutamate in and around the vicinity of GBM cells has been described previously (22). GBM has been shown to make use of glutamate for a variety of metabolic and signaling processes, in conjunction with the local microenvironment $(2,23,24)$. We performed LC-MS based neurotransmitter quantification from paired cortical and tumor samples, which showed a significant difference between the tumor and cortical samples, marked by high concentrations of glutamate in the tumor $(p<0.001, n=6)$, Fig. 1a-b. In order to investigate the dynamics of tumor glutamate release, we used a neocortical slice model where human tissue sections were cultured for 2 days, followed by GBM inoculation and further cultured for an additional 9 days (Workflow, Fig. 1a). Cortical sections without tumor cells showed concentrations of glutamate $<10 \mu \mathrm{M}$ which is consistent with literature for the healthy cortex $(22,25,26)$. Culture medium collected from GBM injected sections showed a significantly 
increased concentration of released glutamate $(470.6 \pm 257.6 \mu \mathrm{M}$, Fig. 1c). These findings are in agreement with experiments carried out in tumor cell culture, where the glutamate concentration was approximately $300 \mu \mathrm{M}$, throughout the culture period. This glutamate level was found to be rapidly restored to values seen in long term culture, reaching a plateau concentration of $273.7 \pm 21.6 \mu \mathrm{M}$ within $<60$ seconds, as has been previously reported in the case of astrocytes, Fig. 1d. These dynamics are confirmed to be due to the large intracellular glutamate stores, which was significantly higher than measured extracellularly (extra- vs. intracellular; 295.2-325.8 $\mu$ M vs. 599.7-695.0 $\mu \mathrm{M} ; \mathrm{p}<0.0001$ ), Supp. Fig. 1a. This remarkable variation between cell lines which was observed in our GBM model is less prominent in in vitro cultures, suggesting that the neural microenvironment plays a major role in regulating glutamate homeostasis.

These high levels of extracellular glutamate have been reported to be beneficial in the maintenance of tumor cell viability by reduction of reactive oxygen species (ROS, in hypoxic regions), through cysteineglutamate exchange (24), Fig. 1e. The glutamate antiporter xCT exports intracellular glutamate in conjunction with cysteine import. To validate the role of $\mathrm{xCT}$ in glutamate release, we made use of an $\mathrm{xCT}$ inhibitor, Sulfasalazine (SAS, $500 \mu \mathrm{M})(27,28)$, which revealed a significant reduction of extracellular glutamate ( $70 \mu \mathrm{M}$ vs. $\sim 400 \mu \mathrm{M}, \mathrm{p}<0.00001)$. Additionally, we observed that SAS treatment results in a significant reduction in proliferation $(p=0.021, n=3)$ and increase in apoptosis, Supp. Fig. 1b-c, Fig 1f-h. These findings suggest that the XCT could play a significant role in cellular viability within hypoxic regions of the tumor. To investigate the spatial metabo-transcriptomic architecture within hypoxic regions, we made use of a recently published dataset, showing a significant enrichment of cysteine metabolism within hypoxic regions (29), Fig. 1i. Gene expression of System xCT (Gene: SLC7A11) was pronounced within the reactive subtype of GBM Fig $1 \mathrm{j}$. Administration of hypoxia $\left(3 \% \mathrm{O}_{2}\right)$ to primary cell cultures confirmed an increased release of glutamate, not observed in artificial hypoxia $\left(\mathrm{CoCl}_{2}\right)$, Fig. 1k. Further treatment with a common chemotherapeutic (Temodal, TMZ) also revealed a significant increase in extracellular glutamate levels, suggesting that stress induced by toxicity leads to increased glutamate release, Fig. 11. Therefore, we hypothesize that glutamate release might also play a role as an autocrine stimulator to help mitigate the effects of toxic stress.

\section{Rapid enrichment of the local micro-environment with cytotoxic levels of glutamate:}

To further study the effects of autocrine stimulation by secreted glutamate, we estimated the cellular kinetics due to glutamate stimulation, which revealed an increased migratory behavior in stimulated cells $(n=100, p=0.001$, Fig. 2a-b, Supp. Fig. 2a-d). Glutamate stimulation also led to increased proliferation $(p=0.035$, Fig. $2 c$, Supp. Fig 2a). Characterization of the expression of Glutamatergic receptors in publicly available transcriptomic data (29) from GBM revealed that AMPA receptors are restricted to cells within the developmental subtype, whereas NMDA receptors were expressed within all lineage states. Exploration of the Metabotropic glutamate receptors revealed a weak expression of Group III receptors, with Group I and Group II receptors preferentially expressed within the developmental niche, Fig 2d, Supp. Fig 2b. Modulation of ionotropic receptors showed some impact on cell proliferation, in particular, antagonism of the AMPA receptors (NBQX, $50 \mu \mathrm{M})$ showed no significant difference in proliferation and 
migration $(p=0.087)$, whereas antagonism of NMDA receptors (AP5, $50 \mu \mathrm{M})$ resulted in significant reduction in cellular proliferation and migration ( $p<0.01)$, Fig. 2e. Inhibition of the metabotropic Group I receptors (mGluR1 and mGluR5) results in a significant reduction of cell proliferation ( $<<0.001)$, Fig. $2 f$. Conversely, treatment with an agonist of Group I receptors resulted in a significant increase in cell proliferation, above what is seen with glutamate stimulation alone ( $p=0.042)$, Fig. $\mathbf{2 g}$. Inhibition of metabotropic group II/III receptors (LY341495, 60nM) showed no effect on cell proliferation ( $p=0.75)$, Fig $2 \mathrm{~h}$. In order to target the autocrine mechanisms leading to cellular survival and resistance, we aimed to identify a receptor group that was not directly required for cellular viability but rather disables cellular response to toxic stress. Our data suggests that the metabotropic Group II/III are not crucial for cellular survival but might play an important role in evading cellular stress. There has been evidence presented that these receptors play an important role in synaptic plasticity, spine maturation and circuit development (30), all of which have been reported to be crucial for GBM growth in the brain (2). Additionally, there have been previous reports detailing the inhibition of mGLuR3 (Group II receptor) sensitizing GBM cells to chemotherapeutic agents in in-vitro models (31).

\section{mGluR3 receptor expression in GBM}

To investigate the role of GRM3 expression in glioblastoma samples, we profiled paired samples obtained from 12 patients, both de novo and recurrent WHO Grade IV Glioblastoma for mGluR3 expression, Fig. 3a. mGluR3 expression was found to be significantly increased in recurrent samples, compared to de-novo tumor samples $(n=12, p<0.0001)$, Fig. 3 b. Higher overall survival was seen in patients with low expression of mGluR3, with increased expression observed more frequently in patients suffering from recurrent glioblastoma, negatively correlated with the overall survival, Fig. 3c. All recurrent tumor samples were acquired from patients that underwent a combined radio and chemotherapy protocol (STUPP treatment) (32). These clinical findings, in combination with our data strengthened the relation between increased mGluR3 expression as a mechanism of chemoresistance. Additionally, regional heterogeneity in mGluR3 expression was observed, with mGluR3 ${ }^{+}$cells preferentially observed in the leading-edge zone, Fig. $\mathbf{3 d}$. Highest expression of mGluR3 $^{+}$cells was observed along the leading edge of the glioblastoma, which is consistent with transcriptomic data, with GRM3 expression predominantly in the neurodevelopmental subtype of GBM, Fig 2d. Similar results were reported in the GlioVis data portal (33), with mGLuR3 (GRM3) expression most pronounced in the infiltrating region of the tumor, Supp. Fig 3a.

\section{mGluR3 inhibition leads to changes in gene expression, but does not affect survival and cellular kinetics}

We induced mGluR3 inhibition by means of LY341495, a highly potent and selective antagonist for Group II metabotropic glutamate receptors, at nanomolar concentrations ( $60 \mathrm{nM}$, Fig. 4a). Inhibition of mGluR3 neither affected GBM cell proliferation nor cell viability in cell culture, as well as morphology, Fig. $\mathbf{2 h} \mathbf{\mathbf { 4 }} \mathbf{\mathbf { b }} \mathbf{\mathbf { d }} \mathbf{d}$, Supp. Fig. 4a-b. Quantification of cellular kinetics shows that there is no significant difference in cellular movement and all other measured features, Fig. 4e, Supp. Fig 4c-f. To gain insights into the transcriptional effects of inhibition, we performed microarray-based transcriptomic analysis. Top 100 most downregulated genes were related to pathways involving cell junction assembly and cell-cell 
adhesion, Fig. 4f. GSEA analysis shows that there was a significant downregulation of pathways related to neurite growth (GO: regulation of neuron projection regeneration, negative regulation of nervous system development), Fig. $\mathbf{4 f - h}$. Recent reports have pointed to the presence of multiple cell states within the GBM, with the lineage state representing the cells that actively proliferate and migrate to ensure GBM survival $(29,34)$. We show that GBM cells treated with LY341495 show a downregulation of pathways associated with the neural-like, oligodendrocyte-like and astrocyte-like genes, Supp. Fig. $\mathbf{4 g}$. Additionally, quantification of apoptosis/necrosis in the presence of mGluR3 inhibition confirmed no significant increase in apoptotic/necrotic cells, Supp. Fig. 4h. Taken together, we show that mGluR3 inhibition has no direct cytotoxic impact on GBM cells, however, resulting in crucial transcriptional changes that could be relevant in light of additional cell-stressing influences such as alkylating chemotherapeutics.

\section{mGluR3 inhibition facilitates TMZ-induced cytotoxicity}

Since we present a role of mGluR3 receptor expression and a resistance against alkylating chemotherapeutics, we aimed to overcome chemoresistance by mGluR3 inhibition. Only the combination of mGluR3 inhibition (LY341495) and TMZ showed significant antiproliferative and cytotoxic effects throughout different cell lines $(n=3)$, whereas singular application of TMZ was ineffective. The effect could be potentiated by increasing the dosage of TMZ in the combination therapy from 50 to $100 \mu \mathrm{M}$, Fig. 5a-b. Additionally, cellular activity was significantly reduced in all three GBM cell lines under the combinatorial treatment with TMZ and LY341495, Fig 5c, Supp. Fig 5a-c. Temporal analysis of apoptosis lead to precisely monitored cytotoxic effects of the combination treatment. Fluorescence imaging revealed that the combination of TMZ $(25-50 \mu \mathrm{M})$ plus LY341495 $(60 \mathrm{nM})$ was exclusively able to induce cell apoptosis within a short period of time (10-240 min), Fig. 5d. All profiled cell lines showed high sensitivity to the combination treatment, marked by the dramatic increase of early apoptotic pSIVA ${ }^{+}$cells throughout the treatment. Further, quantifying late apoptotic/dying $\mathrm{Pl}^{+}$cells, confirmed the finding that initiation of early apoptosis by TMZ plus mGluR3 inhibition was not just transient, but rather caused definitive cell death in the vast majority of cells, Supp. Fig. 5d-e. Geneset enrichment analysis of samples treated with TMZ vs TMZ+LY341495 show a suppression of pathways related to nucleosome assembly, DNA packaging complex and nucleosome organization, Fig. $\mathbf{5 e}$.

Due to the fact that in vitro models are limited in recapitulating the complexity of glioblastoma within their neural environment, we made use of our recently established human organotypic section-based GBM model, $(11,12,29,35)$. We inoculated GBM cells into cultured human cortical organotypic sections. Due to the usage of a known unmethylated TMZ resistant cell line (12), we did not find increased cell death by TMZ treatment alone, but rather increased growth to escape cytotoxicity and sustain clonal growth (36). The combination of LY341495 and TMZ limited tumor growth within 2 days of treatment, significantly reducing the total tumor area increase over time, Fig. $\mathbf{5 f - g}$. Subsequent immunostaining revealed that the combination treatment resulted in a dramatic alteration of tumor morphology within the GBM model, Fig $5 \mathrm{~h}$.

\section{Discusssion}


Glutamatergic signaling is crucial in the maintenance, proliferation and invasive behavior of GBM. Several studies have investigated the capability of excessive paracrine glutamate signaling between neurons and GBM to promote cell proliferation, migration and tumor establishment $(23,24,37-39)$. We present evidence that there is a strong relationship between glutamate release and stressors to the GBM, validated using a spatial multi-omics approach. We found that any alteration of glutamate uptake/release results in significant alteration of GBM behavior, playing an important role in driving proliferation and invasive behavior. lonotropic and metabotropic receptors play varying but isolated roles, with ionotropic receptors playing a major role in cellular migration/ invasion and metabotropic receptors playing a role in proliferation and therapy resistance. In the healthy brain metabotropic group II receptors have been shown to play an important role in synaptic plasticity, spine maturation and circuit development (30). There have been several high-profile reports detailing the synaptic integration of GBM into neural circuits to promote tumor proliferation and invasion $(1,2)$. Therefore, it would be reasonable to hypothesize that the expression of these receptors might play a similar role in GBM, which arises solely in the neural environment. We explored the transcriptomic distribution of the metabotropic glutamate receptors in the developmental phase of GBM and identified Group II metabotropic receptors as being highly expressed, with mGLuR3 being an important modulatory receptor. Our results are in line with previous reports, showing that high GRM3 expression in GBM patients correlates with poorer survival and limited tumor-free survival (40). Our results further extend the previously reported modulatory role of GRM3 $(31,41)$, by treatment using a minimally toxic mGLUr3 antagonist, LY341495, effective at nanomolar concentrations. Although mono treatment with this antagonist had no apparent cytotoxic effects in cell culture, except those that were detected as changes in the transcriptional programs, where the cells exhibited a loss of both lineage and reactive transcriptional programs, represented as loss of neuron-like, astrocyte-like and oligodendrocyte-like signatures. Additionally, we report a downregulation of pathways that are important in the growth and network formation of GBM. It has been previously shown that GBM cells need this network forming ability to be able to resist chemotherapeutic assault (42). Therefore, by disrupting this ability to function as a singular entity, it is possible to increase susceptibility to therapy. Our results are in agreement with this hypothesis, where we saw that the efficacy of TMZ as a treatment against GBM was significantly potentiated when coupled with LY341495.

In our work, we provide proof of concept of the idea that subtle changes in the transcriptional programs of the tumor, which might not affect the apparent viability of the GBM, when coupled with standard therapy, could lead to significant enhancement of therapeutic efficacy, even in therapy resistant GBM. By targeting these particular transcriptional features, we show that the GBM cells lose their inherent ability to resist chemotherapeutic agents, leading to increased efficacy even in therapy resistant tumors.

Additionally, our work demonstrates that some changes in the GBM cannot be recapitulated within cell culture and is better represented within a natural neural environment aptly mimicked by the presented human cortical tissue based GBM model, paving the way towards personalized GBM therapy.

\section{Declarations}

\section{Conflict of Interest}


The authors declare no conflict of interest.

\section{Ethics Statement}

All patient derived sample collection was approved by the local ethics committee and conducted in accordance to the Helsinki declaration. All tissue donors gave written consent for tissue use for academic research.

\section{Acknowledgements}

Elements from figures 1 and 4 were created using BioRender.com. We thank Stella Maria Carro for her support and the provision of her laboratory facilities and equipment.

\section{Funding}

UGH, KJ and VMR were supported by the FMT project, funded by BMBF (Bundes Ministerium für Bildung and Forschung), (Project Number: 13GW0230A). DHH is funded by the Else Kröner-Fresenius Foundation and spatial transcriptomic work was carried out as part of the MEPHISTO project, funded by BMBF (Project Number: 031L0260B). AW and NS was supported by DFG (Project number: SFB824C04).

\section{Author Contributions}

$\mathrm{KJ}, \mathrm{JPM}, \mathrm{VMR}, \mathrm{SPB}, \mathrm{JvE}, \mathrm{JMG}$ and NG carried out experiments and data acquisition. JK developed celltracer and carried out analysis. VMR and PW carried out spatial sequencing data acquisition and analysis. AKW carried out spatial metabolomics data acquisition and analysis. LH carried out mass spectroscopy measurements and analysis. DP oversaw and assisted with microarray sequencing. MF oversaw and assisted with Incucyte measurements and analysis. DHH and JK analyzed the spatial transcriptomic and metabolomic data. VMR, DHH and $\mathrm{KJ}$ conceived the presented idea, carried out data curation and analysis. JPM, KJ and VMR wrote the initial draft of the manuscript. UGH, JB, OS helped oversee the project and provided resources. All authors discussed the results and were involved in the preparation of the final submission draft.

\section{References}

1. Venkataramani V, Tanev DI, Strahle C, Studier-Fischer A, Fankhauser L, Kessler T, et al. Glutamatergic synaptic input to glioma cells drives brain tumour progression. Nature. 2019 Sep 18;573(7775):5328.

2. Venkatesh HS, Morishita W, Geraghty AC, Silverbush D, Gillespie SM, Arzt M, et al. Electrical and synaptic integration of glioma into neural circuits. Nature. 2019 Sep 18;573(7775):539-45.

3. Ishiuchi S, Yoshida Y, Sugawara K, Aihara M, Ohtani T, Watanabe T, et al. Ca2+-permeable AMPA receptors regulate growth of human glioblastoma via Akt activation. J Neurosci. $2007 \mathrm{Jul}$ 25;27(30):7987-8001. 
4. Stepulak A, Rola R, Polberg K, Ikonomidou C. Glutamate and its receptors in cancer. J Neural Transm. 2014 Aug;121(8):933-44.

5. Stepulak A, Luksch H, Gebhardt C, Uckermann O, Marzahn J, Sifringer M, et al. Expression of glutamate receptor subunits in human cancers. Histochem Cell Biol. 2009 Oct;132(4):435-45.

6. Brocke KS, Staufner C, Luksch H, Geiger KD, Stepulak A, Marzahn J, et al. Glutamate receptors in pediatric tumors of the central nervous system. Cancer Biol Ther. 2010 Mar 15;9(6):455-68.

7. Buckingham SC, Campbell SL, Haas BR, Montana V, Robel S, Ogunrinu T, et al. Glutamate release by primary brain tumors induces epileptic activity. Nat Med. 2011 Sep 11;17(10):1269-74.

8. Snyder EM, Philpot BD, Huber KM, Dong X, Fallon JR, Bear MF. Internalization of ionotropic glutamate receptors in response to mGluR activation. Nat Neurosci. 2001 Nov;4(11):1079-85.

9. Ong WY, He Y, Tan KK, Garey LJ. Differential localisation of the metabotropic glutamate receptor mGluR1a and the ionotropic glutamate receptor GluR2/3 in neurons of the human cerebral cortex. Exp Brain Res. 1998 Apr;119(3):367-74.

10. Heiland DH, Gaebelein A, Börries M, Wörner J, Pompe N, Franco P, et al. Microenvironment-Derived Regulation of HIF Signaling Drives Transcriptional Heterogeneity in Glioblastoma Multiforme. Mol Cancer Res. 2018 Jan 12;16(4):655-68.

11. Henrik Heiland D, Ravi VM, Behringer SP, Frenking JH, Wurm J, Joseph K, et al. Tumor-associated reactive astrocytes aid the evolution of immunosuppressive environment in glioblastoma. Nat Commun. 2019 Jun 11;10(1):2541.

12. Ravi VM, Joseph K, Wurm J, Behringer S, Garrelfs N, Errico P d', et al. Human organotypic brain slice culture: a novel framework for environmental research in neuro-oncology. Life Sci Alliance. 2019 Aug;2(4):e201900305.

13. Wurm J, Behringer SP, Ravi VM, Joseph K, Neidert N, Maier JP, et al. Astrogliosis Releases ProOncogenic Chitinase 3-Like 1 Causing MAPK Signaling in Glioblastoma. Cancers (Basel). 2019 Sep $26 ; 11(10)$.

14. Xu H, Wang Z, Zhu L, Sui Z, Bi W, Liu R, et al. Targeted Neurotransmitters Profiling Identifies Metabolic Signatures in Rat Brain by LC-MS/MS: Application in Insomnia, Depression and Alzheimer's Disease. Molecules. 2018 Sep 17;23(9).

15. Behringer S, Wingert V, Oria V, Schumann A, Grünert S, Cieslar-Pobuda A, et al. Targeted metabolic profiling of methionine cycle metabolites and redox thiol pools in mammalian plasma, cells and urine. Metabolites. 2019 Oct 18;9(10).

16. Piccinini F, Kiss A, Horvath P. CellTracker (not only) for dummies. Bioinformatics. 2016 Mar 15;32(6):955-7.

17. Schindelin J, Arganda-Carreras I, Frise E, Kaynig V, Longair M, Pietzsch T, et al. Fiji: an open-source platform for biological-image analysis. Nat Methods. 2012 Jun 28;9(7):676-82.

18. Heynckes S, Gaebelein A, Haaker G, Grauvogel J, Franco P, Mader I, et al. Expression differences of programmed death ligand 1 in de-novo and recurrent glioblastoma multiforme. Oncotarget. 2017 Sep 26;8(43):74170-7. 
19. Therneau TM, Grambsch PM. Modeling Survival Data: Extending the Cox Model. New York, NY: Springer New York; 2000.

20. Love MI, Huber W, Anders S. Moderated estimation of fold change and dispersion for RNA-seq data with DESeq2. Genome Biol. 2014;15(12):550.

21. Yu G, Wang L-G, Han Y, He Q-Y. clusterProfiler: an R package for comparing biological themes among gene clusters. OMICS. 2012 May;16(5):284-7.

22. Ye ZC, Sontheimer H. Glioma cells release excitotoxic concentrations of glutamate. Cancer Res. 1999 Sep 1;59(17):4383-91.

23. Sontheimer $\mathrm{H}$. Malignant gliomas: perverting glutamate and ion homeostasis for selective advantage. Trends Neurosci. 2003 Oct;26(10):543-9.

24. Sontheimer $\mathrm{H}$. A role for glutamate in growth and invasion of primary brain tumors. $J$ Neurochem. 2008 Apr;105(2):287-95.

25. de Groot J, Sontheimer H. Glutamate and the biology of gliomas. Glia. 2011 Aug;59(8):1181-9.

26. Mahmoud S, Gharagozloo M, Simard C, Gris D. Astrocytes Maintain Glutamate Homeostasis in the CNS by Controlling the Balance between Glutamate Uptake and Release. Cells. 2019 Feb 20;8(2).

27. Gout PW, Buckley AR, Simms CR, Bruchovsky N. Sulfasalazine, a potent suppressor of lymphoma growth by inhibition of the $x(c)$ - cystine transporter: a new action for an old drug. Leukemia. 2001 Oct;15(10):1633-40.

28. Chung WJ, Sontheimer $\mathrm{H}$. Sulfasalazine inhibits the growth of primary brain tumors independent of nuclear factor-kappaB. J Neurochem. 2009 Jul;110(1):182-93.

29. Ravi VM, Will P, Kuekelhaus J, Sun N, Joseph K, Salie H, et al. Spatiotemporal heterogeneity of glioblastoma is dictated by microenvironmental interference. BioRxiv. 2021 Feb 17;

30. Edfawy M, Guedes JR, Pereira MI, Laranjo M, Carvalho MJ, Gao X, et al. Abnormal mGluR-mediated synaptic plasticity and autism-like behaviours in Gprasp2 mutant mice. Nat Commun. 2019 Mar 29;10(1):1431.

31. Ciceroni C, Arcella A, Mosillo P, Battaglia G, Mastrantoni E, Oliva MA, et al. Type-3 metabotropic glutamate receptors negatively modulate bone morphogenetic protein receptor signaling and support the tumourigenic potential of glioma-initiating cells. Neuropharmacology. 2008 Sep;55(4):568-76.

32. Stupp R, Mason WP, van den Bent MJ, Weller M, Fisher B, Taphoorn MJB, et al. Radiotherapy plus concomitant and adjuvant temozolomide for glioblastoma. N Engl J Med. 2005 Mar 10;352(10):987-96.

33. Bowman RL, Wang Q, Carro A, Verhaak RGW, Squatrito M. GlioVis data portal for visualization and analysis of brain tumor expression datasets. Neuro Oncol. 2017;19(1):139-41.

34. Neftel C, Laffy J, Filbin MG, Hara T, Shore ME, Rahme GJ, et al. An integrative model of cellular states, plasticity, and genetics for glioblastoma. Cell. 2019 Aug 8;178(4):835-849.e21.

35. Ravi VM, Neidert N, Will P, Joseph K, Kuekelhaus J, Vollmer L, et al. Lineage and Spatial Mapping of Glioblastoma-associated Immunity. BioRxiv. 2020 Jun 2; 
36. Banelli B, Carra E, Barbieri F, Würth R, Parodi F, Pattarozzi A, et al. The histone demethylase KDM5A is a key factor for the resistance to temozolomide in glioblastoma. Cell Cycle. $2015 \mathrm{Nov}$ 2;14(21):3418-29.

37. Lyons SA, Chung WJ, Weaver AK, Oguninu T, Sontheimer H. Autocrine glutamate signaling promotes glioma cell invasion. Cancer Res. 2007 Oct 1;67(19):9463-71.

38. Schunemann DP, Grivicich I, Regner A, Leal LF, de Araújo DR, Jotz GP, et al. Glutamate promotes cell growth by EGFR signaling on U-87MG human glioblastoma cell line. Pathol Oncol Res. 2010 Jun;16(2):285-93.

39. Rzeski W, Turski L, Ikonomidou C. Glutamate antagonists limit tumor growth. Proc Natl Acad Sci USA. 2001 May 22;98(11):6372-7.

40. Ciceroni C, Bonelli M, Mastrantoni E, Niccolini C, Laurenza M, Larocca LM, et al. Type-3 metabotropic glutamate receptors regulate chemoresistance in glioma stem cells, and their levels are inversely related to survival in patients with malignant gliomas. Cell Death Differ. 2013 Mar;20(3):396-407.

41. Zhou K, Song Y, Zhou W, Zhang C, Shu H, Yang H, et al. mGlu3 receptor blockade inhibits proliferation and promotes astrocytic phenotype in glioma stem cells. Cell Biol Int. 2014 Apr;38(4):426-34.

42. Osswald M, Jung E, Sahm F, Solecki G, Venkataramani V, Blaes J, et al. Brain tumour cells interconnect to a functional and resistant network. Nature. 2015 Dec 3;528(7580):93-8.

\section{Tables}


Table 1. List of reagents, supplier and product numbers

\begin{tabular}{|c|c|c|}
\hline Reagent & Supplier & $\begin{array}{l}\text { Product } \\
\text { number }\end{array}$ \\
\hline D-Serine & Sigma & S4250-5G \\
\hline L-Serine & Sigma & S4500-1G \\
\hline Dopamine & Sigma & $\mathrm{H} 8502-5 \mathrm{G}$ \\
\hline Dopamine-D4 hydrochloride solution & Sigma & D-072-1ML \\
\hline Serotonine hydrochloride & Sigma & $\begin{array}{l}\text { H9523- } \\
100 M G\end{array}$ \\
\hline GABA, $\gamma$-amino butyric acid & Sigma & A2129-10G \\
\hline L-Glutamate & Sigma & 49621-250G \\
\hline Glycine hydrochloride & Sigma & G2879-100G \\
\hline Glycine $-1-{ }^{13} \mathrm{C},{ }^{15} \mathrm{~N}$ & Sigma & $\begin{array}{l}\text { 299340- } \\
100 \mathrm{MG}\end{array}$ \\
\hline L-2-Aminoadipic acid & Sigma & $\begin{array}{l}\text { A7275- } \\
250 M G\end{array}$ \\
\hline Tryptophan & Sigma & T8941-25G \\
\hline Isoprenaline & Sigma & I5627-5G \\
\hline Water, CHROMASOLV ${ }^{\mathrm{TM}}$ LC-MS & $\begin{array}{l}\text { Honeywell Research } \\
\text { Chemicals }\end{array}$ & 39253-1L \\
\hline $\begin{array}{l}\text { Methanol, CHROMASOLV'M LC-MS Ultra, tested for } \\
\text { UHPLC-MS }\end{array}$ & $\begin{array}{l}\text { Honeywell Research } \\
\text { Chemicals }\end{array}$ & $14262-1 \mathrm{~L}$ \\
\hline AC $3 \mathrm{AQ}$ column $1.0 \times 150 \mathrm{~mm}$ & HiChrom & $\begin{array}{l}\text { ACE-116- } \\
1501\end{array}$ \\
\hline
\end{tabular}


Table 2. Mass transitions and optimized mass spectrometry parameters for the neurotransmitters and internal standards in this study.

\begin{tabular}{llllllll} 
Q1 & Q3 & Dwelling time $(\mathrm{ms})$ & Metabolite & DP & EP & CE & CXP \\
\hline 177,1 & 160,2 & 20 & Serotonine $(5-H T)$ & 38 & 10 & 10 & 7 \\
\hline 148,2 & 84,1 & 20 & Glutamate (Glu) & 41 & 10 & 24 & 15 \\
\hline 104,2 & 87,1 & 20 & GABA (g-aminobutyric acid) & 26 & 14 & 15 & 16 \\
\hline 154,1 & 137,2 & 20 & Dopamine (DA) & 37 & 10 & 13 & 8 \\
\hline 205,2 & 188 & 20 & Tryptophan & 40 & 4 & 14 & 12 \\
\hline 182,2 & 136,1 & 20 & Tyrosine & 52 & 8 & 25 & 7 \\
\hline 212 & 152,2 & 20 & Isoprenaline & 46 & 8 & 20 & 9 \\
\hline 76,1 & 30 & 20 & Glycine (Gly) & 6 & 7,6 & 19 & 14 \\
\hline 158,1 & 141,2 & 20 & D4-Dopamine & 37 & 10 & 13 & 8 \\
\hline 106,1 & 60 & 20 & Serine (Ser) & 6 & 10,5 & 15,5 & 7 \\
\hline 78,1 & 31 & 20 & 13C, ${ }^{15} \mathrm{~N}-$ Gly & 76 & 10 & 39 & 8 \\
\hline 105,048 & 59,1 & 20 & Choline & 56 & 10 & 21 & 10
\end{tabular}

Figures 
a.

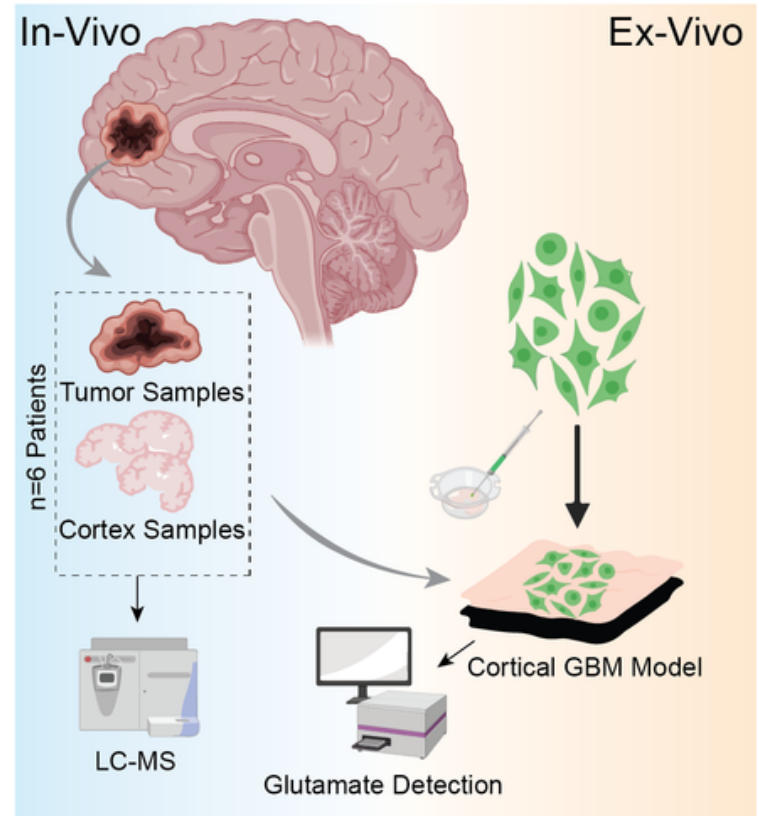

f.

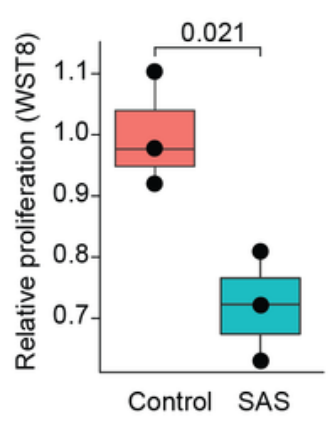

g.

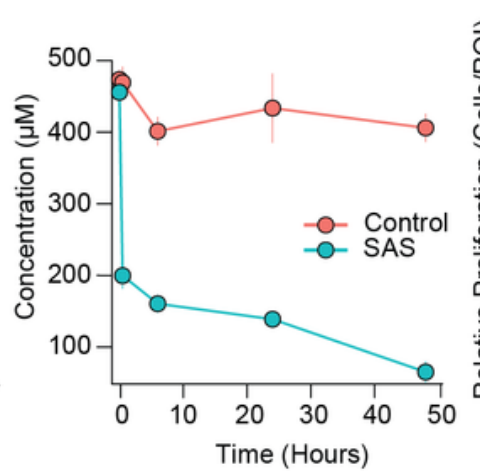

b.

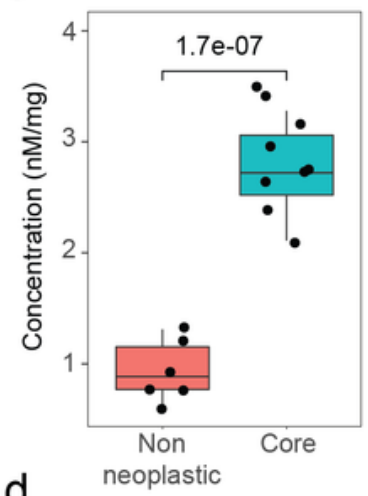

d.

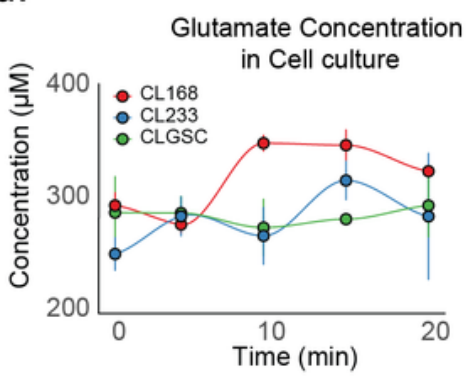

c.

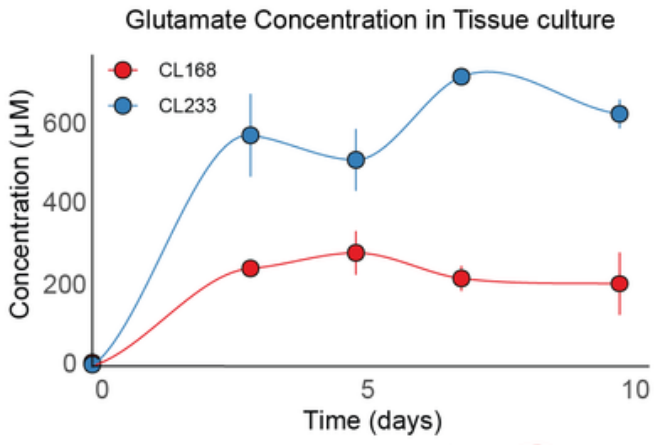

e.

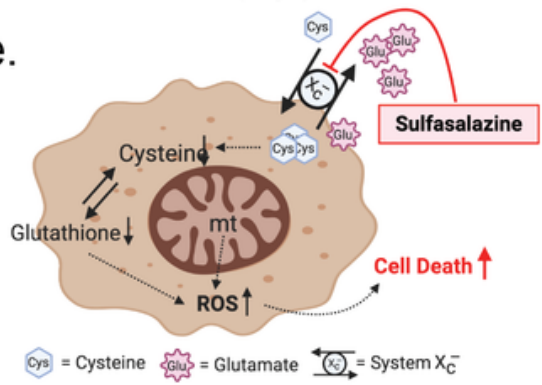

i. Hallmark Hypoxia

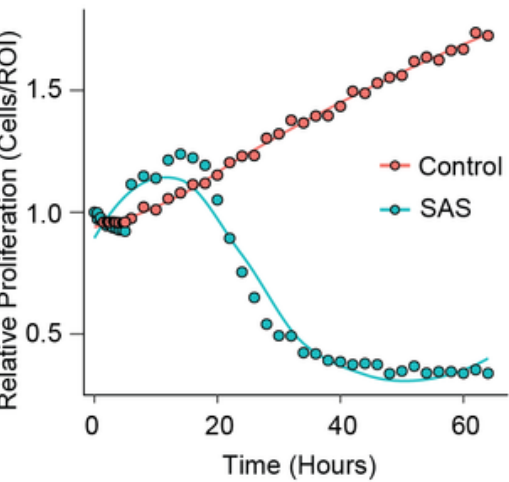

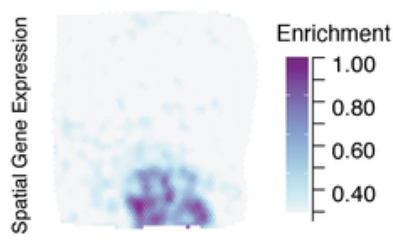

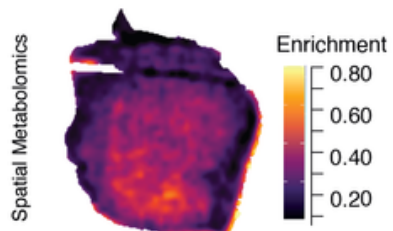

j.

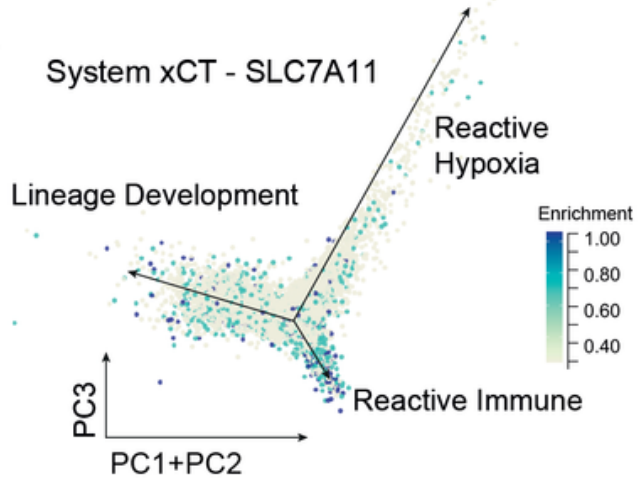

k.

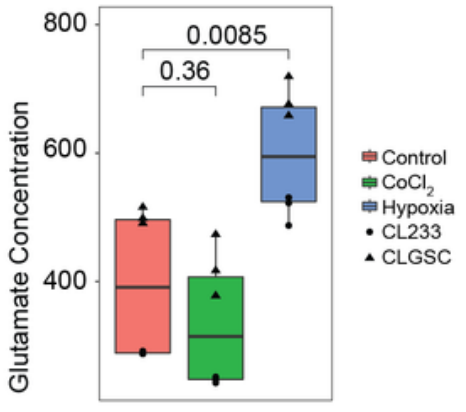

I.

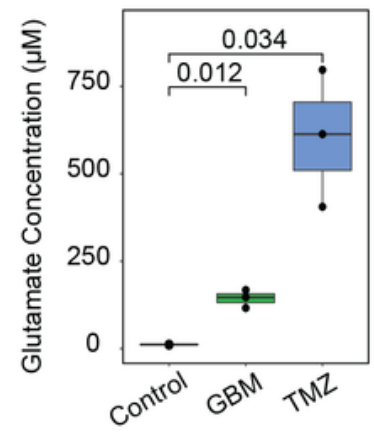

Figure 1

Glutamate dynamics in GBM. (a) Workflow of the experimental paradigm used to study glutamate concentration within a GBM microenvironment. (b) Mass Spectroscopy based measurements of glutamate concentration from tissue samples sourced from the tumor core vs non infiltrated tissue show a significantly higher concentration of glutamate in the core ( $n=6$ samples, $p<0.001$, t-test). (c) Healthy cortical tissue was inoculated with GBM cells and cultured over a period of 9 days. The growth medium 
was collected over the culture period and the glutamate concentration was quantified over a time period of 9 days. (d) Glutamate concentration in cell culture reaches a plateau within one minute of medium swap. The maximum concentration is reached within 10 mins, which then maintained over the measurement period. (e) Mechanism of action of sulfasalazine, where the inhibition of the GlutamateCysteine transporter leads to an increase in ROS within the cells, leading to cell death. (f) Sulfasalazine treatment has a significant impact on Proliferation, as measured by WST-8 proliferation assay $(n=3$, $\mathrm{p}=0.021$ ). (g) Inhibition of the Glutamate/Cysteine symporter by means of Sulfazalazine (500uM) results in a significant reduction in the extracellular glutamate concentration over 48hrs. (h) Relative proliferation of cells treated with SAS shows a significant decrease in comparison to control cells. (i) Spatially resolved expression profile of Hypoxic regions shows an overlap with spatially resolved metabolomics of cysteine metabolism (j) Expression of SLC7A11 within GBM samples show an enrichment within reactive hypoxia subtype of GBM (k) Exposure of GBM to hypoxic conditions result in a significant increase in glutamate concentration $(n=3, p=0.0085)$. This increase in glutamate concentration was not observed in artificial hypoxia, by $\mathrm{CoCl} 2(n=3, p=0.36)$ (I) Glutamate concentration measurements from tissue culture experiments where cortical sections were inoculated with GBM shows a significant increase in comparison to control sections $(n=3, p=0.012)$. When the sections were further treated with $T M Z, a$ significant increase in glutamate concentration was seen with respect to inoculated sections $(n=3$, $\mathrm{p}=0.034$ ). All box plots represent median, with hinges representing 25 th and 75 th percentile and whiskers representing $1.5 x$ Interquartile range.

a

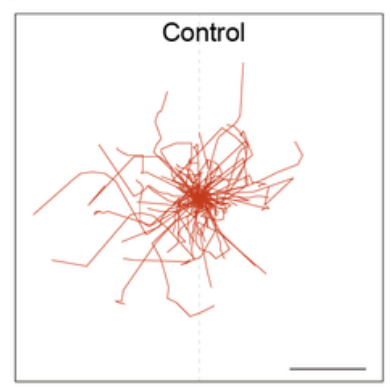

e

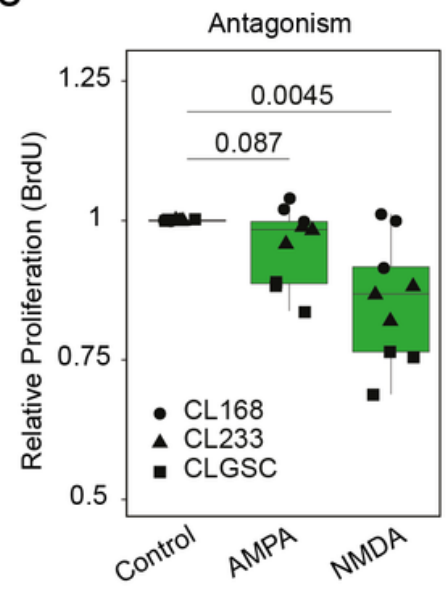

b

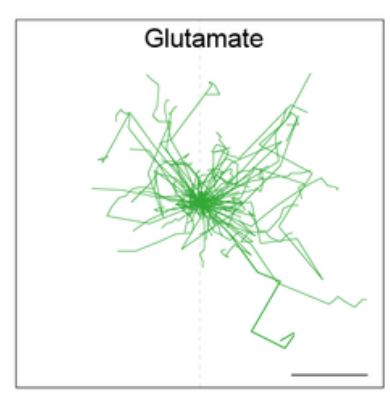

f

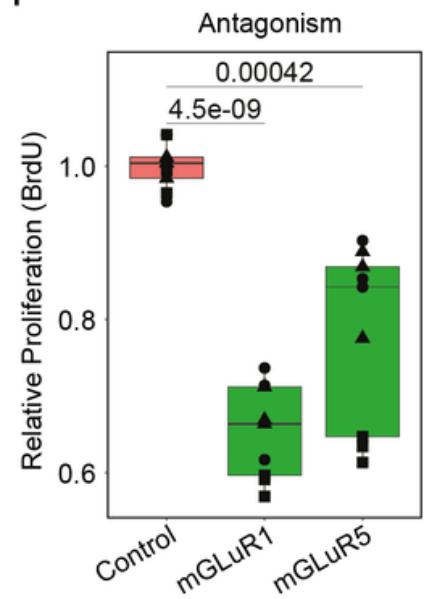

C

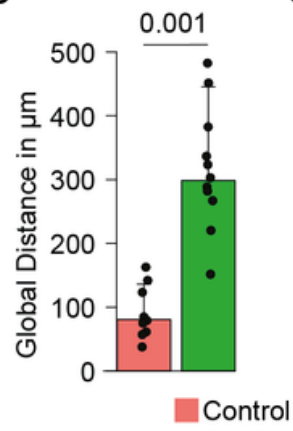

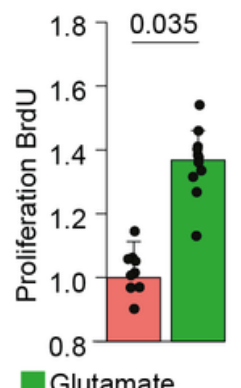

g

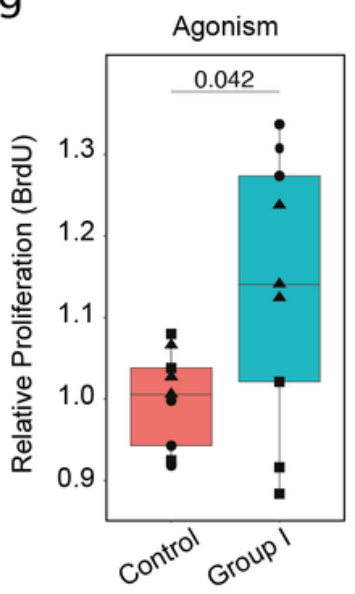

d

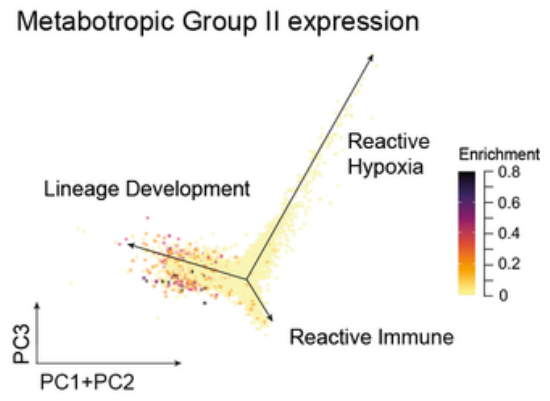

$\mathrm{h}$

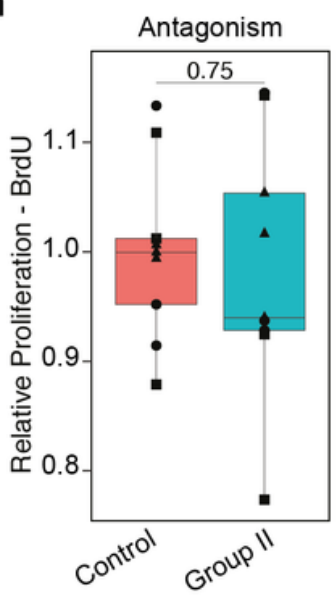

- CL168

- CL233

- CLGSC

Figure 2 
Glutamate receptors and their function in GBM. (a) Glutamate stimulation shows that there is a significant increase in the movement in comparison to unstimulated controls ( $n=24$ cells each). Scale bar $=50 \square \mathrm{m}$ (b) Quantification of the global distance travelled shows that there is a significant increase in the global distance travelled by GBM cells that are stimulated with glutamate vs unstimulated controls $(n=24$ each, $p=0.001)$. (d) Expression of Metabotropic Group II receptors was localized to the lineage subtype of GBM cells, with minimal expression in the reactive subtypes (e) Antagonism of AMPA and NMDA receptors revealed that only the inhibition of NMDA receptors resulted in a decrease in cell proliferation ( $n=9, p=0.0045)$ ( $f$ ) Antagonism of Group I metabotropic receptors (mGLuR1 and mGLuR5) resulted in a significant reduction in cell proliferation $(n=9, p<0.001)(g)$ Agonism of Group I receptors significantly increased the proliferation of GBM $(n=9, p<0.05)(h)$ Agonism of Group II metabotropic receptors show no change in proliferation $(n=9, p>0.05)$. All box plots represent median, with hinges representing 25th and 75th percentile and whiskers representing $1.5 x$ Interquartile range. 
a
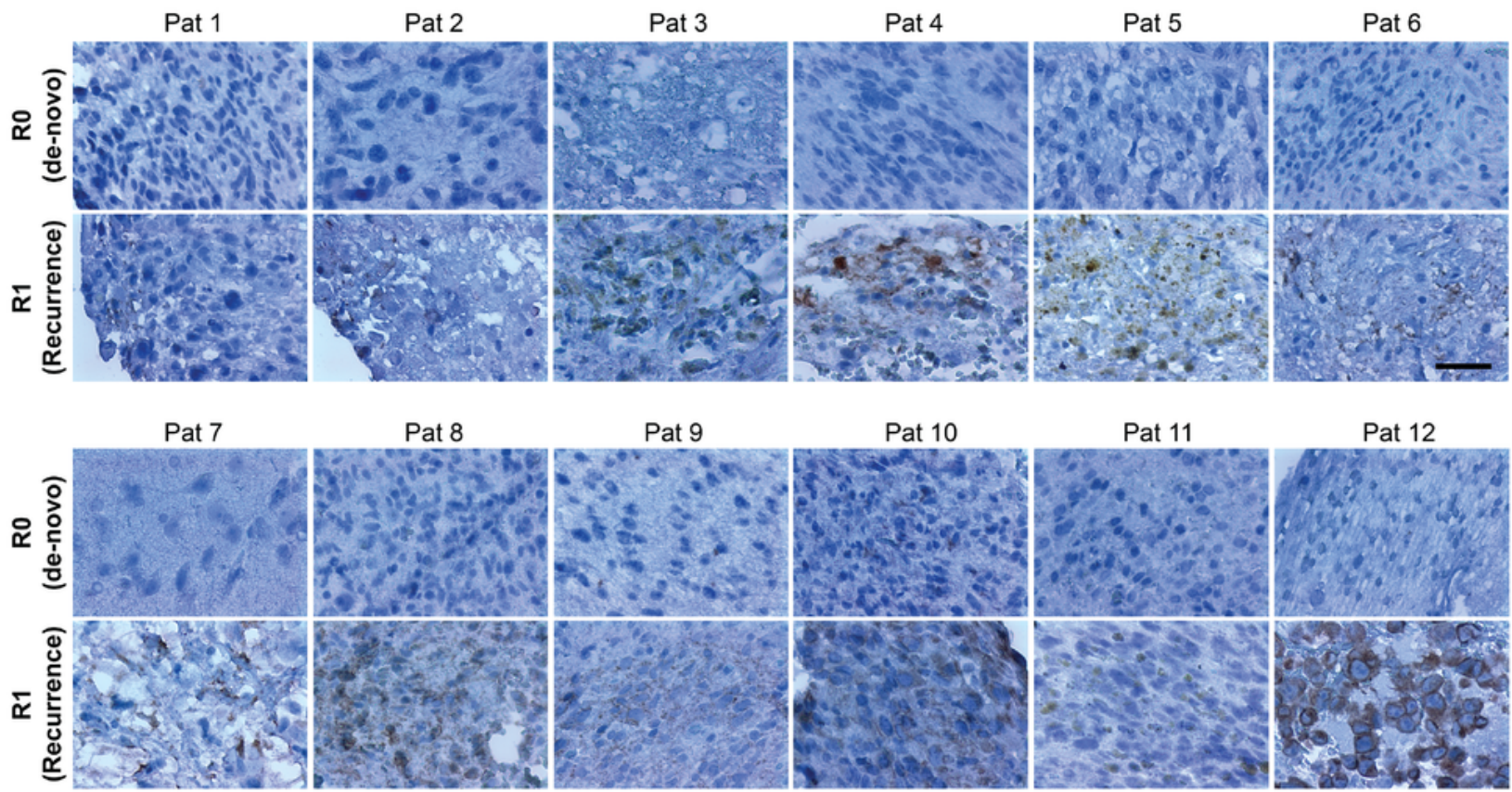

b

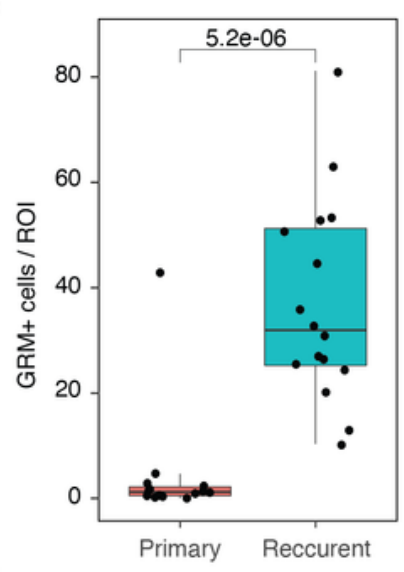

d

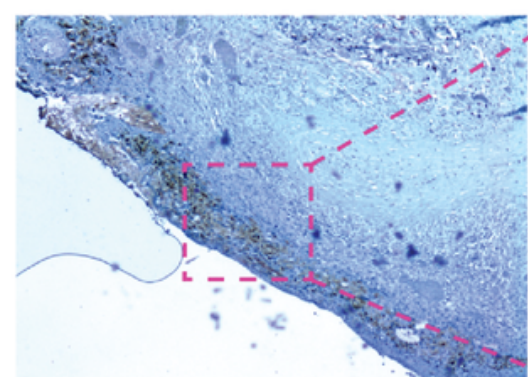

$5 x$
C
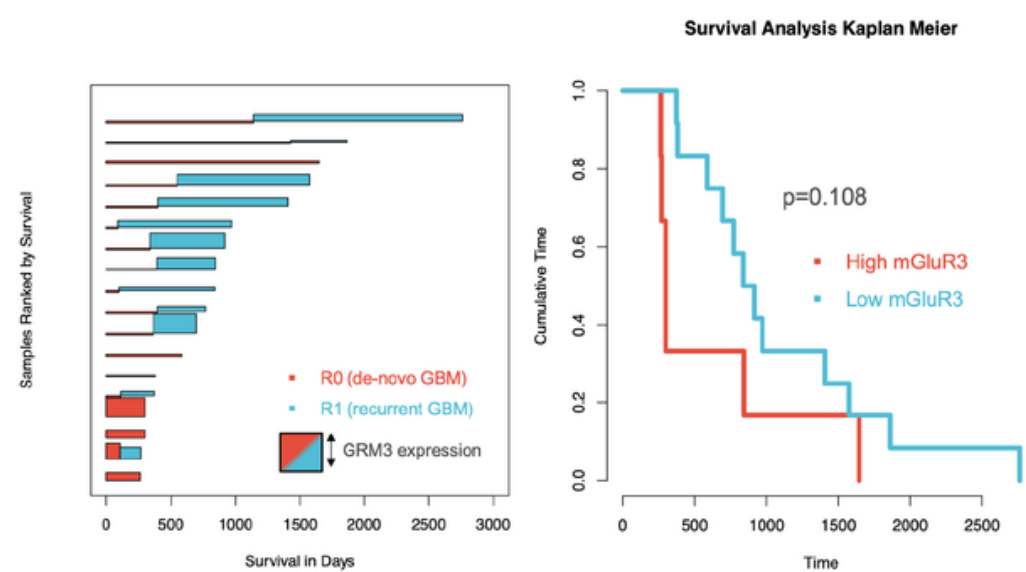

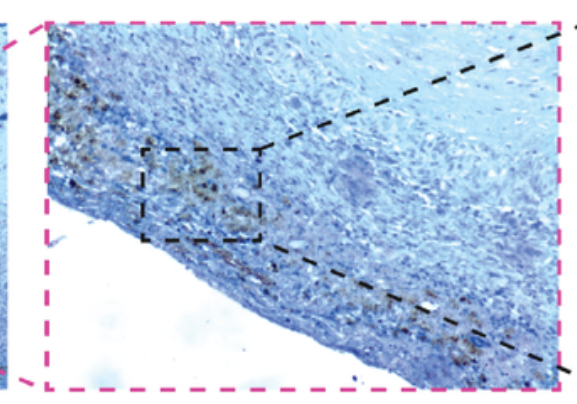

$10 \mathrm{x}$

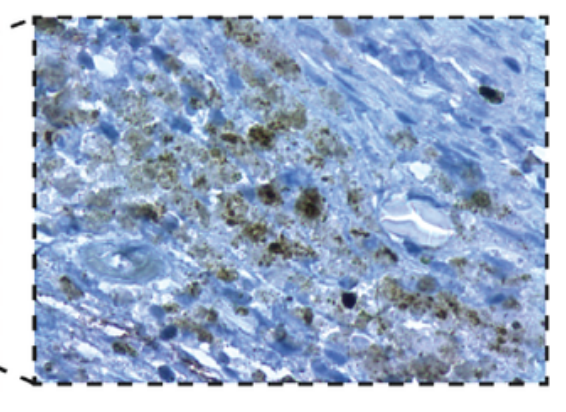

$40 \mathrm{x}$

\section{Figure 3}

GRM3 expression in GBM patients. (a) Patient derived samples show that there is a significant increase in GRM3+ cells in recurrent patients. (b) Quantification of GRM3+ cells within the samples show that there is a significant increase in the number of cells in the recurrent samples in comparison to de-novo samples ( $\mathrm{n}=18$ patients, 12 paired samples). Scale bar is $100 \mu \mathrm{m}$ (c) Survival analysis from the patient cohort reveals that there is a trend towards increased patient survival when there are fewer GRM3+cells within 
the tumor. (d) Magnified image of a patient sample containing GRM3+ cells. Box plot represents median, with hinges representing 25th and 75th percentile and whiskers representing $1.5 x$ Interquartile range.

a.
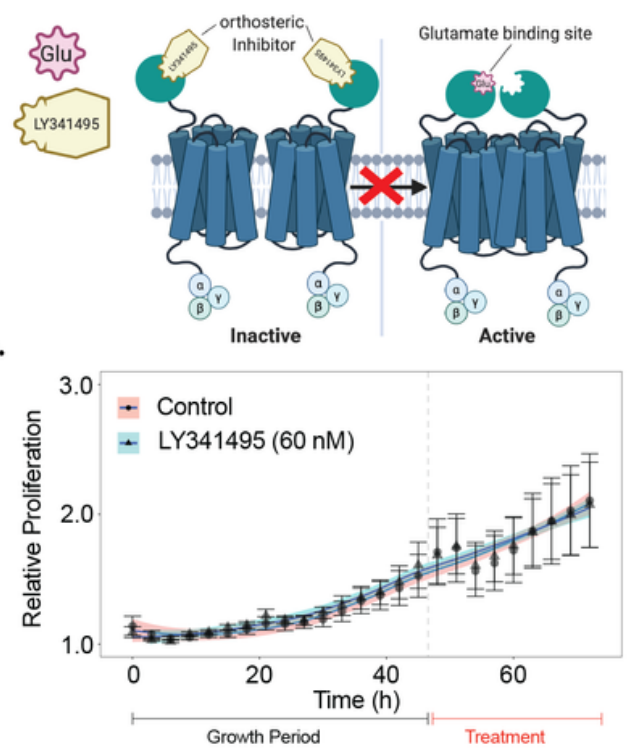

f.

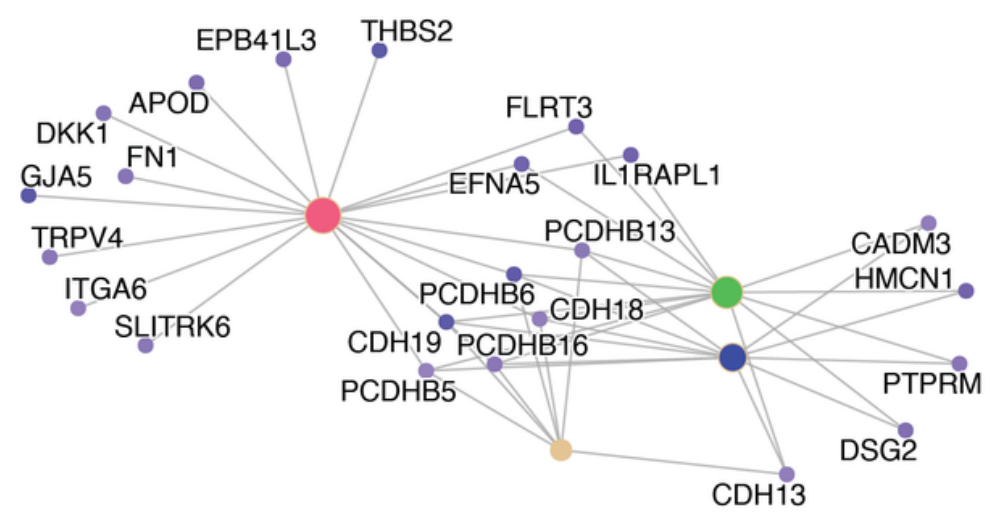

d.

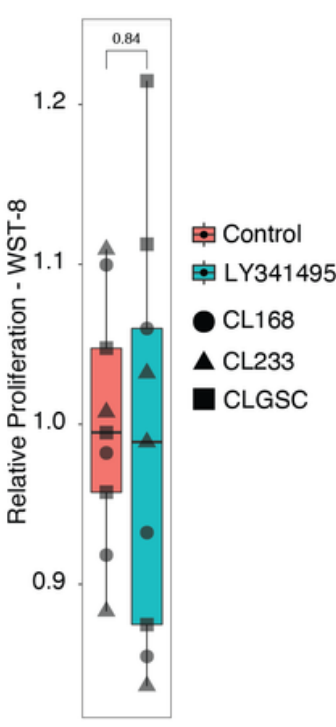

e.

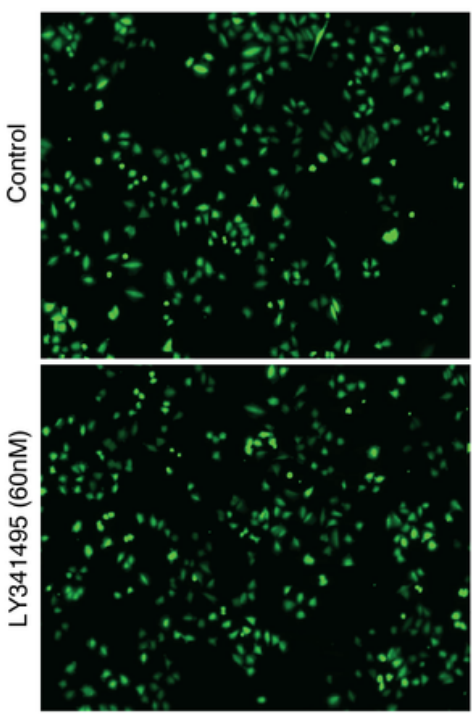

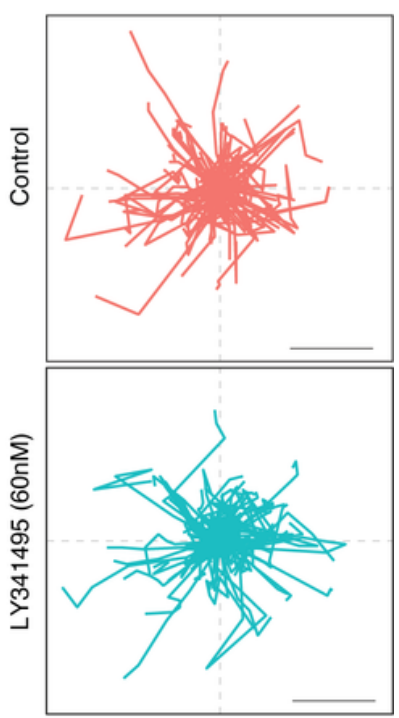

g.

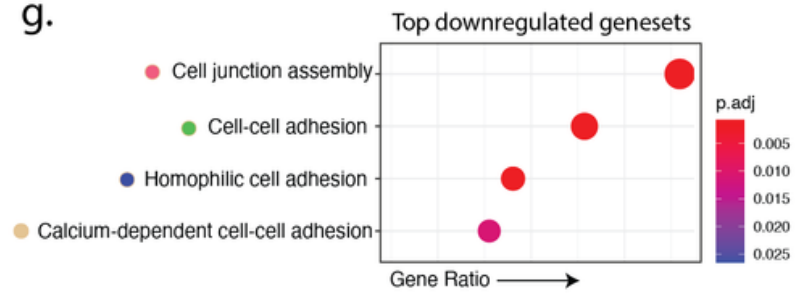

h.

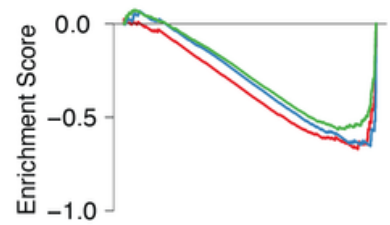

Regulation of Axonogenesis (p.adj $=0.0007$ ) Regulation of neuron projection regeneration $($ p.adj $=0.0045$ ) Regulation of neuron precursor cell proliferation $(p . a d j=0.007$ )

\section{Figure 4}

The effect of mono-therapeutic stimulation using LY341495 (a) Mechanism of action of LY341495. LY341495 is a competitive antagonist for the mGluR3 receptor. (b) Live imaging experiments carried out over a $72 \mathrm{hr}$ time period shows that there is no difference in proliferation as a result of the treatment with LY341495. (c) Stimulation with LY341495 shows no change in proliferation, measured using WST-8 ( $n=9$, $\mathrm{p}=0.94$ ). (d) Representative Images showing that there is no change in cell viability as a result of mGLuR3 inhibition by LY341495 (e) Quantification of cellular kinetics. Time-lapse imaging was carried out over 72 hours and features pertaining to movement were extracted from $n=100$ cells per condition. Migration plots from cells that were treated with LY341495 versus unstimulated controls shows no difference in the distance travelled ( $\mathrm{n}=100$ each). Scale bar is $50 \mu \mathrm{m}$ (f) Transcriptomic analysis shows a significant reduction in expression of genes related to cell junction assembly and cell-cell adhesion. (g) Geneset enrichment analysis shows a downregulation of pathways related to cellular adhesion and tumor formation (h) Geneset Enrichment Analysis shows a significant downregulation of neural related 
pathways. All box plots represent median, with hinges representing 25th and 75th percentile and whiskers representing 1.5x Interquartile range.

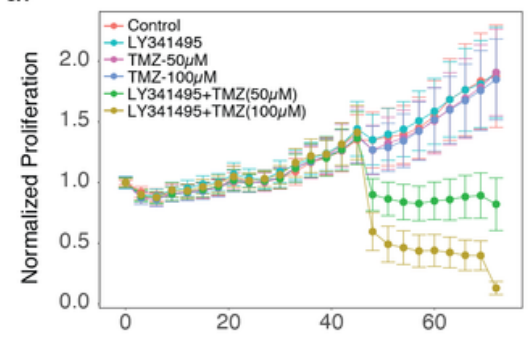

d.

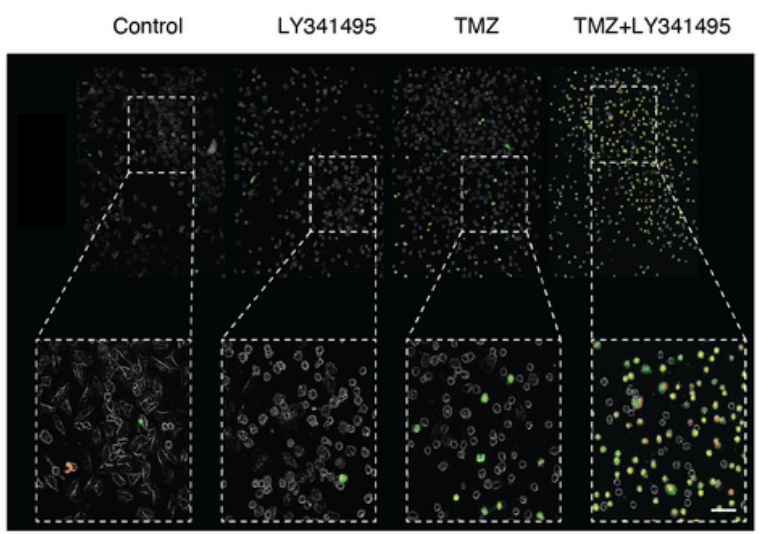

f.

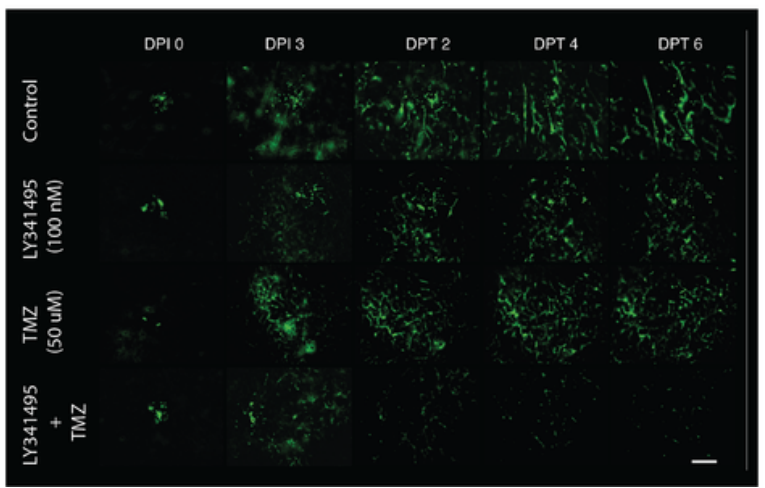

b.

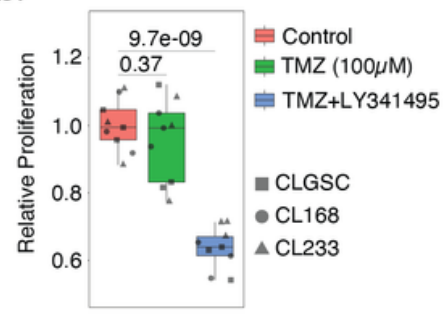

c.

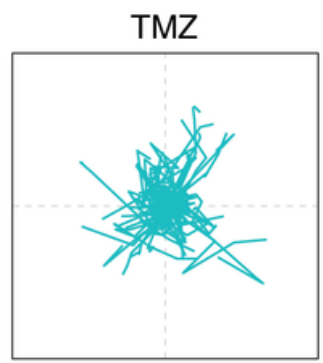

TMZ+LY341495

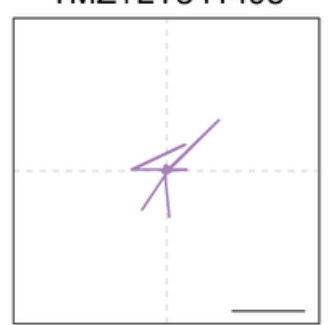

e.

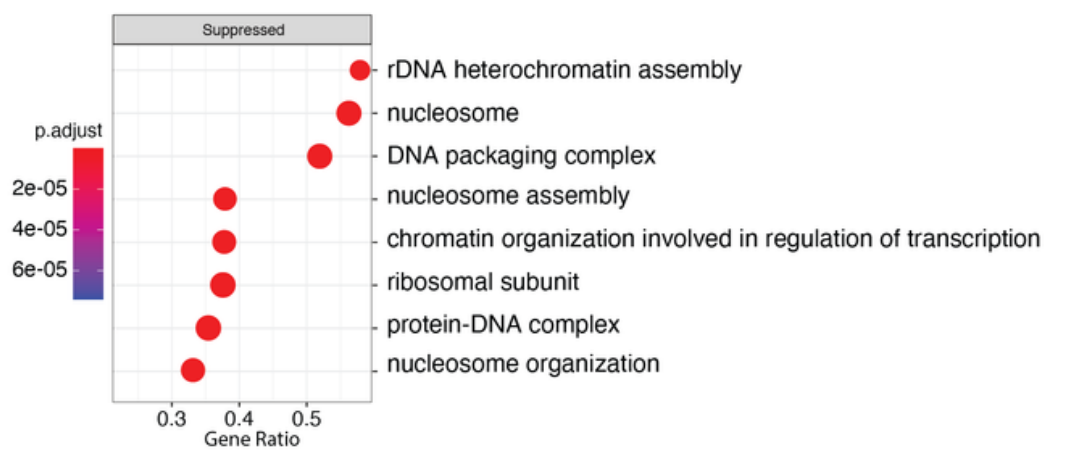

g.

h.
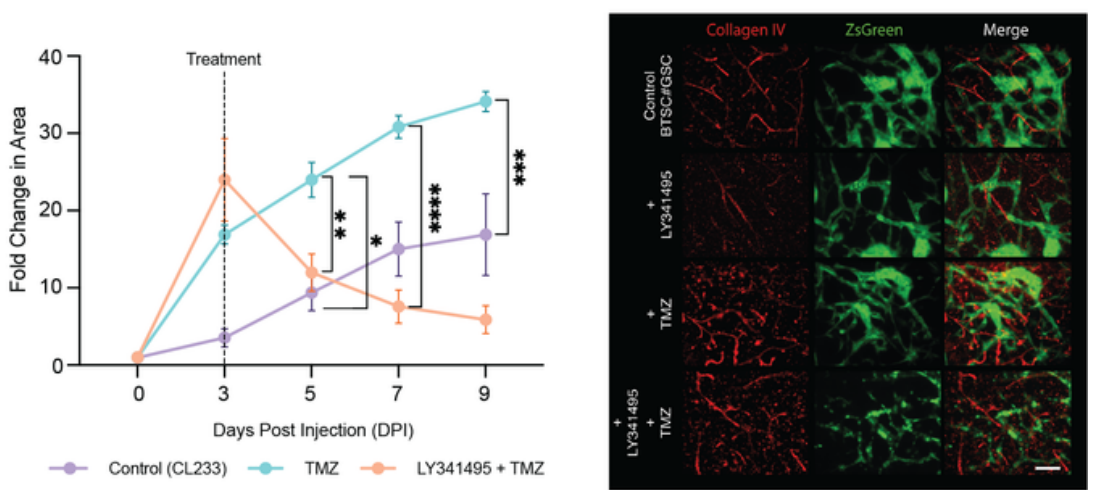

\section{Figure 5}

Efficacy of neo-adjuvant therapy with GRM3 inhibition in experimental conditions. With TMZ resistant cell lines (a) Quantification of time lapse imaging experiments show that TMZ alone had minimal effects of cellular proliferation. When combined with LY341495, there is a significant reduction in proliferation. The effect is potentiated when the dosage of TMZ is increased to $100 \mu \mathrm{M}$. (b) Relative proliferation measured by BrdU shows that there is no change in proliferation as a result of treatment with $\operatorname{TMZ}(n=3, p>0.05)$, whereas there is a significant decrease in proliferation when cells were treated with the combined approach $(p<0.0001)$ (c) Quantification of cellular migration shows that there is a significant reduction in cellular migration as a result of the combination therapy (d) Kinetic apoptosis measurement. Cells in early apoptosis bind to pSIVA, showing green fluorescence. When the cells are necrotic, PI is able to bind to the nuclear membrane, leading to red fluorescence. Kinetic apoptosis assay shows that the combined treatment is the most effective in inducing terminal PI+ cells within 4 hours of treatment. The cells treated 
with the combination therapy appear yellow due to the expression of both pSIVA (green) and PI (red). Scale bar is $50 \square \mathrm{m}$ (e) Geneset enrichment analysis from samples treated with TMZ vs. TMZ+LY341495 (f) Growth profiles of GBM model sections under different treatment conditions. The combination treatment showed the most effectivity in reducing tumor growth. Scale bar is $250 \square \mathrm{m}(\mathrm{g})$ Quantification of tumor growth over time shows that there is a significant reduction in the tumor growth in the combined treatment strategy. There is no reduction in tumor growth in control sections and TMZ treated $(50 \mu \mathrm{M})$ sections post treatment, whereas a strong reduction in tumor area is seen in the sections treated with the combination therapy. (h) Immunostainings of collagen within the injected slices show GBM cells growing along blood vessels in control, LY341495 and TMZ treated conditions. When the combination therapy was used, we see a strong decrease in the GBM cell growth. Scale bar is $50 \square \mathrm{m}$. The box plot represents median, with hinges representing 25th and 75 th percentile and whiskers representing $1.5 x$ Interquartile range

\section{Supplementary Files}

This is a list of supplementary files associated with this preprint. Click to download.

- SupplementaryMaterialGRM3.docx

- Supp.Fig.1.png

- Supp.Fig2.png

- Supp.Fig3.png

- Supp.Fig4.png

- Supp.Fig5.png 